\title{
WULFF SHAPE EMERGENCE IN GRAPHENE
}

\author{
ELISA DAVOLI, PAOLO PIOVANO, AND ULISSE STEFANELLI
}

\begin{abstract}
Graphene samples are identified as minimizers of configurational energies featuring both two- and three-body atomic-interaction terms. This variational viewpoint allows for a detailed description of ground-state geometries as connected subsets of a regular hexagonal lattice. We investigate here how these geometries evolve as the number $n$ of carbon atoms in the graphene sample increases. By means of an equivalent characterization of minimality via a discrete isoperimetric inequality, we prove that ground states converge to the ideal hexagonal Wulff shape as $n \rightarrow \infty$. Precisely, ground states deviate from such hexagonal Wulff shape by at most $K n^{3 / 4}+\mathrm{o}\left(n^{3 / 4}\right)$ atoms, where both the constant $K$ and the rate $n^{3 / 4}$ are sharp.
\end{abstract}

\section{INTRODUCTION}

The recent realization of high crystalline quality graphene samples at room temperature can be regarded as a breakthrough in Materials Science and has led to the attribution of the 2010 Nobel Prize in Physics to Geim and Novoselov. The fascinating electronic and mechanical properties of single-atom-thick carbon surfaces are believed to offer unprecedented opportunities for innovative applications, ranging from next-generation electronics to pharmacology, and including batteries and solar cells. New findings are emerging at an always increasing pace and involve thousands of researchers worldwide cutting across Materials Science, Physics, and Chemistry, extending from fundamental science to novel applications.

The stand of the mathematical understanding of graphene is comparably less developed. All available results are extremely recent and concern the modeling of transport properties of electrons in graphene sheets [3, 6, 13, 16, 25, 34, 35], homogenization [8, 33], atomistic-to-continuum passage for nanotubes [14], geometry of monolayers under Gaussian perturbations [11], external charges [27] or magnetic fields [10], combinatorial description of graphene patches [22], and numerical simulation of dynamics via nonlocal elasticity theory [44]. Remarkably, the determination of the equilibrium shapes and the Wulff shapes of graphene samples and graphene nanostructures is still a challenging problem [1, 5, 19].

Graphene ideally corresponds to a regular, two-dimensional, hexagonal arrangement of carbon atoms. In the bulk of a graphene sample each carbon atom is covalently bonded to three neighbors. These covalent bonds are of $s p^{2}$-hybridized type and ideally form $2 \pi / 3$ angles in a plane. In order to describe these bonds, some phenomenological interaction energies, including two- and three-body interaction

2010 Mathematics Subject Classification. 82D25.

Key words and phrases. Ground state, hexagonal lattice, isoperimetric inequality, Wulff shape. 
terms, have been presented and partially validated [38, 39]. The arrangement of carbon atoms in the two-dimensional crystal emerges then as the global effect of the combination of local atomic interactions, and can be seen as the result of a geometric optimization process: by identifying the configuration of $n$ carbon atoms with their positions $\left\{x_{1}, \ldots, x_{n}\right\} \subset \mathbb{R}^{2}$, one minimizes a given configurational energy $E: \mathbb{R}^{2 n} \rightarrow \mathbb{R} \cup\{\infty\}$ and proves that the minimizers are indeed subsets of a regular hexagonal lattice. The configurational energies for carbon feature a decomposition $E=E_{2}+E_{3}$ where $E_{2}$ corresponds to an attractive-repulsive two-body interaction, favouring some preferred spacing of the atoms, and $E_{3}$ encodes three-body interactions, expressing the specific geometry of $s p^{2}$ covalent bonding in carbon.

The above variational viewpoint brings the study of graphene geometries into the realm of the so-called crystallization problems. A first analysis in this direction is in [12], where $E_{2}$ is assumed to be of Lennard-Jones type and $E_{3}$ favors $2 \pi / 3$ and $4 \pi / 3$ bond angles. The focus of [12] is on the thermodynamic limit: as $n \rightarrow \infty$ the minimal energy density is proven to converge to a finite value, corresponding indeed to the configuration in which the atoms arrange themselves in a suitably stretched hexagonal lattice. Analogous thermodynamic-limit results are obtained in [15], where nonetheless the term $E_{2}$ favors $\pi$ bond angles. The crystallization problem for a finite number of carbon atoms is studied in [32] where the periodicity of ground states as well as the exact quantification of the ground-state energy is obtained, together with the discussion of carbon nanostructures such as fullerenes and nanotubes, see also [28, 29, 32]. The reader is referred to [20, 41, 42] for onedimensional crystallization results, to $[24,36,43]$ and [40] in the two-dimensional case either in the finite and in the thermodynamic-limit case, and to [30,31] for crystallization in the square lattice. Results on the three-dimensional thermodynamic limit are available in $[17,18]$, and a recent review on the crystallization problem can be found in [4].

Our analysis moves from the consideration that, as the configurational energy favors bonding, ground states are expected to have minimal perimeter, since boundary atoms have necessarily less neighbors. This heuristics is here made precise by providing a new characterization of ground states based on a crystalline isoperimetric inequality. Indeed, we prove in Proposition 3.4 below that ground states correspond to isoperimetric extremizers and we determine the exact isoperimetric constant. Analogous results are obtained in $[30,31]$ for the square lattice, and in [9] for the triangular lattice.

The minimality of the ground-state perimeter gives rise to the emergence of large polygonal clusters as the number of atoms $n$ increases. Indeed, one is interested in identifying a so-called Wulff shape to which all properly rescaled ground states converge. This has been successfully obtained for both the triangular [2, 9, 37] and the square lattice $[30,31]$, where ground states approach a hexagon and a square, respectively, as $n \rightarrow \infty$. Quite remarkably, in both the triangular and the square case it has been proved that ground states differ from the Wulff shape by at most $\mathrm{O}\left(n^{3 / 4}\right)$ atoms, this bound being sharp. This is what it is usually referred to as the $n^{3 / 4}$-law.

The central aim of this paper is to establish the Wulff shape emergence for graphene samples and to investigate the $n^{3 / 4}$-law in this setting. Precisely, we 
provide sharp quantitative convergence results for ground states $G_{n}$ to the correspondingly rescaled Wulff shape, in terms both of the Hausdorff distance and of the flat distance of the empirical measures $\mu_{G_{n}}$, to the measure with density $\frac{4}{3 \sqrt{3}} \chi_{W}$, i.e. the rescaled characteristic function of the (rescaled) hexagonal Wulff shape.

With respect to previous contributions to this subject the novelty of our paper is threefold. First, we provide a complete characterization of ground states, for all numbers of atoms, as well as a detailed description of their geometry. In particular, as a byproduct of our isoperimetric characterization we are able to investigate the edge geometry of graphene patches. Graphene atoms tend to naturally arrange themselves into hexagonal samples whose edges can have, roughly speaking, two shapes: they can either form zigzag or armchair structures (see [5, 21, 26] and below)).

We prove here that hexagonal configurations having armchair edges do not satisfy the isoperimetric equality, whereas those with zigzag edges do (see Definition 4.1). Namely, we have the following.

Theorem 1.1 (Zigzag-edge selectivity). Zigzag hexagons are ground states, armchair hexagons are not.

This provides an analytical counterpart to the experimental results in [26], confirming the zigzag-edge selectivity in the growth process of graphene samples.

The second main result of the paper is the discussion of the Wulff shape emergence in the hexagonal system, which is not a simple Bravais lattice but rather a so-called multilattice. We relate the Wulff shape emergence with the isoperimetric nature of ground states. Our result reads as follows.

Theorem 1.2 (Emergence of the Wulff shape). Let $G_{n}$ be a sequence of ground states in the hexagonal lattice. Let $W_{n}$ be the zigzag hexagon centered in the origin and with side $r_{n}$ (see (5) below). Then, there exists a suitable translation $G_{n}^{\prime}$ of $G_{n}$ such that

$$
\left|G_{n}^{\prime} \backslash W_{n}\right| \leq K n^{3 / 4}+\mathrm{o}\left(n^{3 / 4}\right)
$$

where $|\cdot|$ is the cardinality of the set, and

$$
K:=\frac{2^{7 / 4}}{3^{1 / 4}}
$$

In addition, there holds

$$
\begin{aligned}
& d_{\mathcal{H}}\left(G_{n}^{\prime}, W_{n}\right) \leq \mathrm{O}\left(n^{1 / 4}\right), \\
& \left\|\mu_{G_{n}^{\prime}}-\mu_{W_{n}}\right\| \leq K n^{-1 / 4}+\mathrm{o}\left(n^{-1 / 4}\right), \\
& \mu_{G_{n}^{\prime}} \rightarrow^{*} \frac{4}{3 \sqrt{3}} \chi_{W} \quad \text { weakly } \text { in the sense of measures, }
\end{aligned}
$$

and

$$
\left\|\mu_{G_{n}^{\prime}}-\frac{4}{3 \sqrt{3}} \chi_{W}\right\|_{\mathrm{F}} \leq K n^{-1 / 4}+\mathrm{o}\left(n^{-1 / 4}\right),
$$

where $d_{\mathcal{H}}$ is the Hausdorff distance, $\|\cdot\|$ is the total variation, and $\|\cdot\|_{F}$ is the flat norm (see (9)). 
Our third main result concerns the sharpness of the $n^{3 / 4}$-law (1). We show not only the sharpness of the convergence ratio, but also of the constant $K$ in front of the leading term. We have the following.

Theorem 1.3 (Sharpness of the $n^{3 / 4}$-law). There exists a sequence of integers $n_{j}$ such that for every sequence of ground states $\left\{G_{n_{j}}\right\}$ properties (1)-(4) hold with equalities.

Our proof strategy differs from that of [30,37], as it is not based on configuration rearrangements. The argument here moves from the control of the radius $r_{G_{n}}$ of the maximal hexagon $H_{G_{n}}$ contained in a ground state $G_{n}$ with $n$ atoms. In particular, we define

$$
r_{n}:=\min \left\{r_{G_{n}}: G_{n} \text { is a ground state with } n \text { atoms }\right\},
$$

and we show that every ground state (up to translation) consists of the $n$-Wulff shape $W_{n}$ with comparably few extra atoms, see Section 6 . Precisely, we prove a delicate estimate of the form $r_{n} \sim n^{1 / 2}$ which entails that the atoms of $G_{n}$ which do not belong to $W_{n}$ are at most $\mathrm{O}\left(n^{3 / 4}\right)$. An outcome of our proof is that the convergence rates and the constants above are sharp. Indeed, we explicitly construct a sequence of integers such that every corresponding sequence of ground states attains the right-hand sides of (1)-(4).

In the triangular lattice, the existence of a sequence of ground states whose deviation from the Wulff shape is exactly of order $n^{3 / 4}$ was exhibited in [37] with no specific control on the convergence constants. With a different implementation of the method discussed here, we revisited the triangular-lattice case in [9], obtaining explicit, sharp convergence constants.

The paper is organized as follows. In Section 2 we introduce some notation and a few definitions. In Section 3 we highlight the isoperimetric nature of ground states. Section 4 contains a discussion of the equilibrium shapes of graphene samples, and a proof of the fact that armchair hexagons are not ground states. In particular we prove there Theorem 1.1. In Section 5 we provide delicate lower and upper bounds for $r_{n}$. Section 6 is eventually devoted to the proofs of Theorems 1.2 and 1.3.

\section{Notation and Setting of the Problem}

Let the hexagonal lattice be given by

$$
\mathcal{L}:=\left\{m t_{1}+n t_{2}+c w: m, n \in \mathbb{Z}, c \in\{0,1\}\right\},
$$

with

$$
t_{1}:=\left(\begin{array}{c}
\sqrt{3} \\
0
\end{array}\right), \quad t_{2}:=\left(\begin{array}{c}
\sqrt{3} / 2 \\
3 / 2
\end{array}\right), \quad \text { and } \quad w:=\left(\begin{array}{l}
0 \\
1
\end{array}\right) .
$$

Note that the minimal distance between points in $\mathcal{L}$ is 1 .

We denote a configuration of $n$ atoms by $C_{n}:=\left\{x_{1}, \ldots, x_{n}\right\} \in \mathbb{R}^{2 n}$, the distance between two atoms, $x_{i}$ and $x_{j}$, by $\ell_{i j}$, and the counterclockwise-oriented angle between the two segments $x_{i}-x_{j}$ and $x_{k}-x_{j}$ by $\theta_{i j k}$. The energy of a configuration 


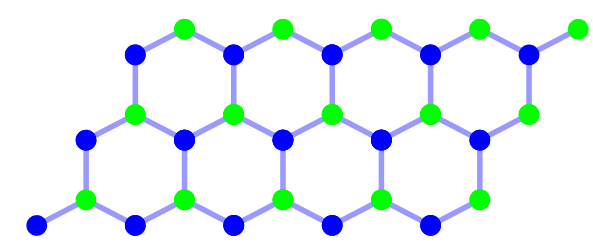

Figure 1. An example of a subset of $\mathcal{L}$.

$C_{n}$ is defined as

$$
E\left(C_{n}\right):=E_{2}\left(C_{n}\right)+E_{3}\left(C_{n}\right)=\frac{1}{2} \sum_{i \neq j} v_{2}\left(\ell_{i j}\right)+\sum_{(i, j, k) \in \mathcal{A}} v_{3}\left(\theta_{i j k}\right)
$$

where $v_{2}:[0, \infty) \rightarrow[-1, \infty]$ and $v_{3}:[0,2 \pi] \rightarrow[0, \infty)$ are the two-body and the three-body interaction potentials. We notice that the energy is invariant under rotations and translations. Two atoms $x_{i}$ and $x_{j}$ are said to be bonded, or there is an (active) bond between $x_{i}$ and $x_{j}$, if $1 \leq \ell_{i j}<\sqrt{2}$. The index set $\mathcal{A}$ in (6) is defined as the set of all triples $(i, j, k)$ for which the angle $\theta_{i j k}$ separates two active bonds. We will always assume that $v_{2}(1)=-1$ and that $v_{2}(\ell)$ vanishes for $\ell \geq \sqrt{2}$ (see below). We work under the assumption that $v_{3}$ reaches its minimum value only at the angles $\pi / 3$ and $2 \pi / 3$, and ground states are subsets of the hexagonal lattice. We use the standard notation for the right- and left-continuous integerparts: $\lfloor x\rfloor:=\max \{z \in \mathbb{Z}: z \leq x\}$ and $\lceil x\rceil:=\min \{z \in \mathbb{Z}: x \leq z\}$, respectively.

Under suitable assumptions on the potentials $v_{2}$ and $v_{3}$, it was established in [32] that all ground-state configurations are (isometric to) subsets of the hexagonal lattice $\mathcal{L}$ and that the value of the energy for every ground state with $n$ atoms is given by

$$
e_{n}:=-\left\lfloor\frac{3 n}{2}-\sqrt{\frac{3 n}{2}}\right\rfloor .
$$

From this point on all configurations are hence seen as subsets of $\mathcal{L}$.

The bond graph of a configuration $C_{n}$ is the graph consisting of all its vertices and active bonds. For every atom $x_{i} \in C_{n}$, we indicate by $b\left(x_{i}\right)$ the number of active bonds of $C_{n}$ with an endpoint in $x_{i}$. Denoting by $B\left(C_{n}\right)$ the total number of bonds in $C_{n}$, there holds

$$
B\left(C_{n}\right)=\frac{1}{2} \sum_{i=1}^{n} b\left(x_{i}\right)
$$

A configuration $C_{n}$ is said to be connected if for every two atoms $y_{1}, y_{2} \in C_{n}$ there exists a collection of atoms $x_{1}, \ldots, x_{i} \in C_{n}$ such that $y_{1}$ is bonded to $x_{1}, x_{i}$ is bonded to $y_{2}$, and every atom $x_{j}$, with $2 \leq j \leq i-1$ is bonded both to $x_{j-1}$ and to $x_{j+1}$. We call minimal simple cycles of a configuration all the simple cycles in the graph that are hexagons of side 1.

The area $A\left(C_{n}\right)$ of a configuration $C_{n}$ is given by the number of minimal simple cycles of $C_{n}$. Denoting by $F\left(C_{n}\right) \subset \mathbb{R}^{2}$ the closure of the union of the regions enclosed by the minimal simple cycles of $C_{n}$, and by $G\left(C_{n}\right) \subset \mathbb{R}^{2}$ the union of all 
bonds which are not included in $F\left(C_{n}\right)$, the perimeter $P$ of a configuration $C_{n}$ is defined as

$$
P\left(C_{n}\right):=\mathcal{H}^{1}\left(\partial F\left(C_{n}\right)\right)+2 \mathcal{H}^{1}\left(G\left(C_{n}\right)\right),
$$

where $\mathcal{H}^{1}$ is the one-dimensional Hausdorff measure. As already observed in [30], there holds

$$
P\left(C_{n}\right)=\lim _{\varepsilon \searrow 0} \mathcal{H}^{1}\left(\partial\left(\partial F\left(C_{n}\right) \cup G\left(C_{n}\right)+B_{\varepsilon}\right)\right)
$$

where $B_{\varepsilon}=\left\{y \in \mathbb{R}^{2}|| y \mid \leq \varepsilon\right\}$.

With a slight abuse of notation, the symbol $|\cdot|$ will denote, according to the context, both the absolute value of a real number and the cardinality of a set.

We will often use the notion of edge boundary $\Theta$ of a configuration with respect to a reference lattice: this is the union of unit segments in the reference lattice that are not included in the graph of $C_{n}$ but share one and only one endpoint with $C_{n}$,

$$
\Theta\left(C_{n}\right):=\left\{(x, y) \in(\mathcal{L})^{2}: x \in C_{n}, y \notin C_{n}\right\} .
$$

The edge perimeter of a configuration $C_{n}$ will be defined as the number of segments belonging to its edge boundary.

For every configuration $C_{n}:=\left\{x_{1}, \ldots, x_{n}\right\}$ in $\mathcal{L}$, we denote by $\mu_{C_{n}}$ the empirical measure associated to the rescaled configuration $\left\{x_{1} / \sqrt{n}, \ldots, x_{n} / \sqrt{n}\right\}$, that is,

$$
\mu_{C_{n}}:=\frac{1}{n} \sum_{i} \delta_{x_{i} / \sqrt{n}}
$$

Given a Lebesgue measurable set $A \subset \mathbb{R}^{2}$, we will designate by $\mathcal{L}^{2}(A)$ its twodimensional Lebesgue measure. For any bounded Radon measure $\mu$, the symbol $\|\mu\|$ will represent its total variation in $\mathbb{R}^{2}$, whereas $\|\mu\|_{F}$ will be the flat norm of $\mu$, defined as

$$
\|\mu\|_{F}:=\sup \left\{\int_{\mathbb{R}^{2}} \varphi d \mu: \varphi \text { is Lipschitz with }\|\varphi\|_{W^{1, \infty}\left(\mathbb{R}^{2}\right)} \leq 1\right\} .
$$

The set of bounded Radon measures on $\mathbb{R}^{2}$ will be denoted by $M_{b}\left(\mathbb{R}^{2}\right)$.

\section{Discrete ISOPERIMETRIC INEQUALITY}

In this section we prove that connected configurations satisfy a discrete isoperimetric inequality, and we characterize ground states as configurations realizing the isoperimetric equality. We first deduce some preliminary relations between the area, the perimeter, the edge perimeter, and the energy of configurations. Let $C_{n}$ be a configuration. Then

$$
E\left(C_{n}\right)=E_{2}\left(C_{n}\right)=-B\left(C_{n}\right)=-\frac{1}{2} \sum_{i=1}^{n} b\left(x_{i}\right) .
$$

Since every atom in $\mathcal{L}$ has exactly 3 bonds, we have

$$
\left|\Theta\left(C_{n}\right)\right|=\sum_{i=1}^{n}\left(3-b\left(x_{i}\right)\right),
$$


and the energy and the edge perimeter of configurations are related by

$$
E\left(C_{n}\right)=-\frac{3}{2} n+\frac{1}{2}\left|\Theta\left(C_{n}\right)\right|
$$

Recalling that every minimal simple cycle of $C_{n}$ consists of 6 bonds, we have

$$
\begin{aligned}
& 6 A\left(C_{n}\right)=2 B\left(C_{n} \cap F\left(C_{n}\right)\right)-B\left(C_{n} \cap \partial F\left(C_{n}\right)\right) \\
& \quad=-2 E\left(C_{n} \cap F\left(C_{n}\right)\right)-\mathcal{H}^{1}\left(\partial F\left(C_{n}\right)\right) .
\end{aligned}
$$

On the other hand,

$$
\mathcal{H}^{1}\left(G\left(C_{n}\right)\right)=B\left(C_{n} \cap G\left(C_{n}\right)\right)=-E\left(C_{n} \cap G\left(C_{n}\right)\right) .
$$

Hence, we obtain

$$
\begin{aligned}
P\left(C_{n}\right)= & \mathcal{H}^{1}\left(\partial F\left(C_{n}\right)\right)+2 \mathcal{H}^{1}\left(G\left(C_{n}\right)\right) \\
= & -2 E\left(C_{n} \cap F\left(C_{n}\right)\right)-6 A\left(C_{n}\right)-2 E\left(C_{n} \cap G\left(C_{n}\right)\right) \\
& =-2 E\left(C_{n}\right)-6 A\left(C_{n}\right),
\end{aligned}
$$

that is

$$
E\left(C_{n}\right)=-3 A\left(C_{n}\right)-\frac{1}{2} P\left(C_{n}\right)
$$

In conclusion, we can express the energy of a hexagonal configuration $C_{n}$ as a linear combination of its area and its perimeter. Likewise, in view of (11), the edge perimeter satisfies

$$
\left|\Theta\left(C_{n}\right)\right|=3 n-6 A\left(C_{n}\right)-P\left(C_{n}\right) .
$$

The following result is a direct corollary of [23, Theorem 7.3, page 142].

Proposition 3.1. There exists a total order $\tau: \mathbb{N} \rightarrow \mathcal{L}$ such that for all $n \in \mathbb{N}$ the configuration $D_{n}$ defined by $D_{n}:=\left\{x_{\tau(1)}, \ldots, x_{\tau(n)}\right\}$ (which we call daisy) minimizes $E$ over all configurations with $n$ atoms, i.e.

$$
E\left(D_{n}\right)=\min _{C_{n} \subset \mathcal{L}} E\left(C_{n}\right)=e_{n}
$$

where $e_{n}$ is the quantity defined in (7).

The total order in Proposition 3.1 is nonunique. For the sake of definiteness we fix here our attention on a specific order $\tau$, as described in [32]. For $n=6 k^{2}$, $k \in \mathbb{N}$, the sequence $\left\{D_{6 k^{2}}\right\}$ is defined inductively as follows: $D_{6}$ is a minimal simple cycle in $\mathcal{L}$, and $D_{24}$ is obtained by externally attaching to all bonds of $D_{6}$ another hexagon. $D_{6 k^{2}}$ is then defined recursively.

For $k, m \in \mathbb{N}$, and $0<m<12 k(k+1), D_{6 k^{2}+m}$ is constructed as in [32, Proof of Proposition 5.1, Step 1]. In view of Proposition 3.1, it is always possible to add one atom to a daisy $D_{n}$ so that the new configuration with $n+1$ points is still a ground state.

To every configuration $C_{n} \subset \mathcal{L}$, we associate a weight function

$$
\Delta_{C_{n}}: C_{n} \rightarrow\{0,1,2\},
$$

defined as

$$
\Delta_{C_{n}}(x):=\left|\left\{y \in C_{n}:(x, y) \in C_{n} \times C_{n}, y<_{\tau} x\right\}\right|,
$$



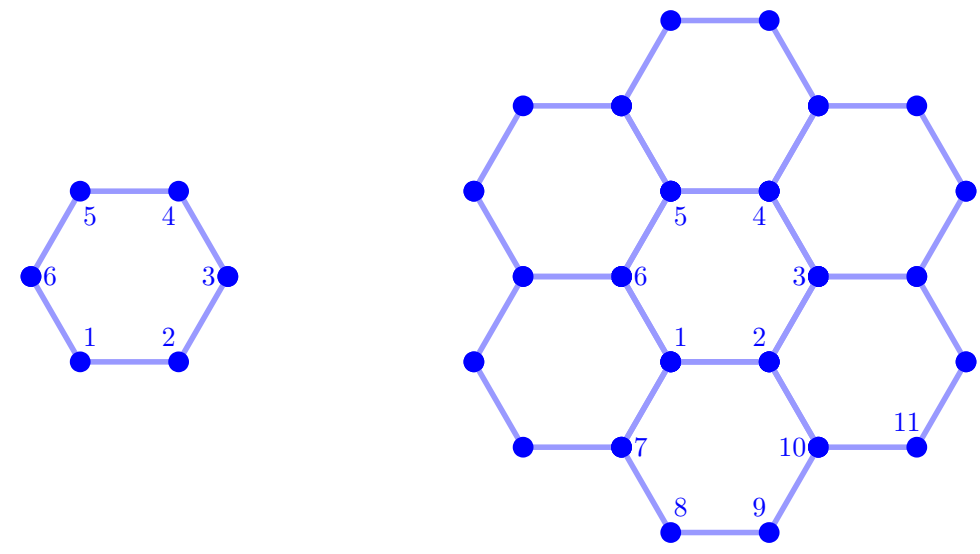

Figure 2. The daisies $D_{6}$ and $D_{24}$ and the total order $\tau$.

for every $x \in C_{n}$, and we rewrite $C_{n}$ as the union

$$
C_{n}=\bigcup_{k=0}^{2} C_{n}^{k}
$$

where

$$
C_{n}^{k}:=\left\{x \in C_{n}: \Delta_{C_{n}}(x)=k\right\}
$$

for $k=0, \ldots, 2$. In particular, $\left|C_{n}^{0}\right|$ corresponds to the number of connected components of $C_{n}$.

The next proposition allows us to express the energy, the perimeter, the edge perimeter, and the area of a configuration $C_{n}$ as a function of the cardinality of the sets $C_{n}^{k}$.

Proposition 3.2. Let $C_{n}$ be a configuration in $\mathcal{L}$. Then,

$$
\begin{aligned}
& E\left(C_{n}\right)=-\left|C_{n}^{1}\right|-2\left|C_{n}^{2}\right|, \\
& A\left(C_{n}\right)=\left|C_{n}^{2}\right|, \\
& P\left(C_{n}\right)=2\left|C_{n}^{1}\right|-2\left|C_{n}^{2}\right|, \\
& \left|\Theta\left(C_{n}\right)\right|=3\left|C_{n}^{0}\right|+\left|C_{n}^{1}\right|-\left|C_{n}^{2}\right|,
\end{aligned}
$$

for every $n>1$. Moreover,

$$
E\left(C_{n}\right)=-3 A\left(C_{n}\right)-\left|\Theta\left(C_{n}\right)\right|+3\left|C_{n}^{0}\right| .
$$

Proof. We first observe that

$$
E\left(C_{n}\right)=-\sum_{i=1}^{n} \Delta_{C_{n}}\left(x_{i}\right)
$$

For $i=0, \ldots, n-1$, let $C_{i}$ be the subset of $C_{n}$ containing its first $i$ points according to the total order $\tau$. If $x_{\tau(i)} \in C_{n}^{0}$, then

$$
A\left(C_{i}\right)-A\left(C_{i-1}\right)=0, \quad P\left(C_{i}\right)-P\left(C_{i-1}\right)=0, \quad\left|\Theta\left(C_{i}\right)\right|-\left|\Theta\left(C_{i-1}\right)\right|=3 ;
$$


if $x_{\tau(i)} \in C_{n}^{1}$, then

$$
A\left(C_{i}\right)-A\left(C_{i-1}\right)=0, \quad P\left(C_{i}\right)-P\left(C_{i-1}\right)=2, \quad\left|\Theta\left(C_{i}\right)\right|-\left|\Theta\left(C_{i-1}\right)\right|=1 ;
$$

whereas, if $x_{\tau(i)} \in C_{n}^{2}$, we have

$$
A\left(C_{i}\right)-A\left(C_{i-1}\right)=1, \quad P\left(C_{i}\right)-P\left(C_{i-1}\right)=-2, \quad\left|\Theta\left(C_{i}\right)\right|-\left|\Theta\left(C_{i-1}\right)\right|=-1 .
$$

Properties (14)-(17) follow from (19)-(21). Claim (18) is a direct consequence of (12), (16), and (17).

In view of Proposition 3.2 we obtain the following.

Proposition 3.3. The following assertions are equivalent and hold true for every connected hexagonal configuration $C_{n}$ :

(i) $\left|\Theta\left(D_{n}\right)\right| \leq\left|\Theta\left(C_{n}\right)\right|$;

(ii) $P\left(D_{n}\right) \leq P\left(C_{n}\right)$;

(iii) $A\left(D_{n}\right) \geq A\left(C_{n}\right)$.

Proof. The first assertion is a direct consequence of (11) and (13), and is equivalent to (ii) by (16) and (17). The equivalence between (ii) and (iii) follows by (12) and (13).

We are now in a position to characterize connected ground states as extremizers of a discrete isoperimetric problem.

Proposition 3.4. Every connected configuration $C_{n}$ satisfies

$$
\sqrt{A\left(C_{n}\right)} \leq k_{n} P\left(C_{n}\right)
$$

where

$$
k_{n}:=\frac{\sqrt{\left\lfloor\left(\alpha_{n}\right)^{2}-\alpha_{n}\right\rfloor-n+1}}{4\left(\alpha_{n}\right)^{2}-4\left\lfloor\left(\alpha_{n}\right)^{2}-\alpha_{n}\right\rfloor-6},
$$

and $\alpha_{n}:=\sqrt{3 n / 2}$.

Moreover, connected ground states correspond to those configurations for which (22) holds with equality, and, equivalently, to those configurations that attain the maximum area

$$
a_{n}:=-n+\left\lfloor\left(\alpha_{n}\right)^{2}-\alpha_{n}\right\rfloor+1
$$

and the minimum perimeter

$$
p_{n}:=4\left(\alpha_{n}\right)^{2}-4\left\lfloor\left(\alpha_{n}\right)^{2}-\alpha_{n}\right\rfloor-6 .
$$

Proof. We claim that

$$
\sqrt{A\left(D_{n}\right)}=k_{n} P\left(D_{n}\right) .
$$

In fact, in view of (14) and Theorem 3.1, there holds

$$
e_{n}=E\left(D_{n}\right)=-\left|D_{n}^{1}\right|-2\left|D_{n}^{2}\right|
$$

whereas by (11) and (17),

$$
3 n+2 e_{n}=\left|\Theta\left(D_{n}\right)\right|=3+\left|D_{n}^{1}\right|-\left|D_{n}^{2}\right|,
$$


where $e_{n}$ is the ground state energy defined in (7). Solving the previous system of equations we deduce

$$
\left|D_{n}^{1}\right|=2 n+e_{n}-2,
$$

and

$$
\left|D_{n}^{2}\right|=-n-e_{n}+1 \text {. }
$$

Claim (24) follows from (15), (16), (25), and (26), by observing that

$$
\begin{aligned}
& \sqrt{A\left(D_{n}\right)}=\sqrt{\left|D_{n}^{2}\right|}=\sqrt{-n-e_{n}+1} \\
& \quad=k_{n}\left(6 n+4 e_{n}-6\right)=k_{n}\left(2\left|D_{n}^{1}\right|-2\left|D_{n}^{2}\right|\right)=k_{n} P\left(D_{n}\right) .
\end{aligned}
$$

Inequality (22) is a direct consequence of (24) and Proposition 3.3. By Proposition 3.3 and (11), connected ground states $G_{n}$ satisfy

$$
\left|\Theta\left(G_{n}\right)\right|=e_{n}+\frac{3}{2} n
$$

and attain the maximum area and the minimum perimeter. The values of $a_{n}$ and $p_{n}$ follow from (17), (16), and (18).

\section{Equilibrium SHAPES OF GRAPHENE SAMPLES}

In this section we characterize the edge geometry of graphene samples. We first introduce a few definitions.

Definition 4.1. For every $s \in \mathbb{N}$ we define the set $\mathcal{H}_{s}^{Z}$ of zigzag hexagons of side $s$ as

$$
\mathcal{H}_{s}^{Z}:=\left\{D_{6 s^{2}}+q: q \in \mathcal{L}\right\}
$$

(for all $s \in \mathbb{N}$, the configuration $D_{6 s^{2}}$ is a complete hexagon of hexagons). For $s \in \mathbb{N}, s \geq 3$, the set $\mathcal{H}_{s}^{A}$ of armchair hexagons of side $s$ is defined as

$$
\mathcal{H}_{s}^{A}:=\left\{A_{s}+q: q \in \mathcal{L}\right\} .
$$

In the expression above, $A_{3}$ is given by the union of $D_{24}$ with 6 extra minimal simple cycles, glued externally to the center of each side of $D_{24}$ (see Figure 3 ). For $s>3, A_{s}$ is defined recursively by adding an extra armchair layer of minimal simple cycles to $A_{s-1}$. We point out that the construction is different for $s$ even and $s$ odd (see Figure 3).

4.1. Proof of Theorem 1.1. The optimality of zigzag hexagons follows in view of Definition 4.1 and Proposition 3.1.

We claim that for every $s \in \mathbb{N}, s \geq 3$, there holds

$$
P\left(A_{s}\right)>p_{\left|A_{s}\right|} .
$$

Indeed, by the definition of $A_{s}$ we have $\left|A_{3}\right|=42$, and for $s>3$

that is

$$
\left|A_{s}\right|=\left|A_{s-1}\right|+ \begin{cases}6(2 s-1) & \text { if } s \text { is even, } \\ 6 s & \text { if } s \text { is odd, }\end{cases}
$$

$$
\left|A_{s}\right|=\frac{9}{2} s^{2}+ \begin{cases}3 s & \text { if } s \text { is even, } \\ \frac{3}{2} & \text { if } s \text { is odd. }\end{cases}
$$




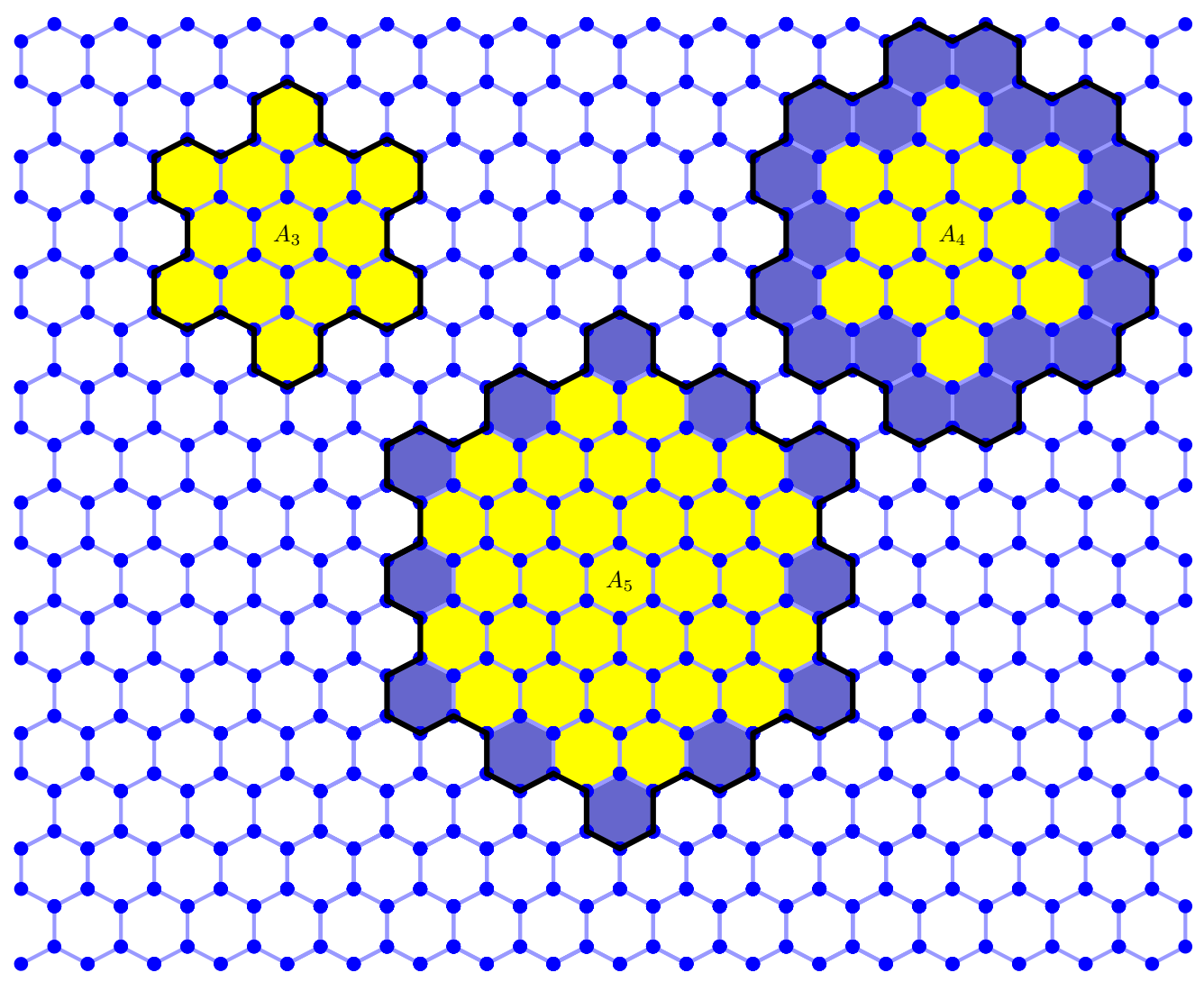

Figure 3 . The armchair hexagons $A_{3}, A_{4}$ and $A_{5}$. Each armchair hexagon $A_{s}$ for $s>3$ is obtained by adding a layer of extra minimal simple cycles (in blue) to the corresponding armchair hexagon $A_{s-1}$ (in yellow). Notice the different structure for $s$ even and for $s$ odd.

On the other hand, the perimeter of each armchair hexagon $A_{s}$ is given by

$$
P\left(A_{s}\right)=12 s-6 .
$$

For $s$ odd, we have

$$
\alpha_{\left|A_{s}\right|}=\sqrt{\frac{3\left|A_{s}\right|}{2}}=\frac{3}{2} s \sqrt{3+\frac{1}{s^{2}}} .
$$

Hence,

$$
\alpha_{\left|A_{s}\right|}^{2}=\frac{9}{4}\left(3 s^{2}+1\right) \in \mathbb{N}
$$

and

$$
\alpha_{\left|A_{s}\right|}^{2}-\left\lfloor\alpha_{\left|A_{s}\right|}^{2}-\alpha_{\left|A_{s}\right|}\right\rfloor=\left\lceil\frac{3}{2} s \sqrt{3+\frac{1}{s^{2}}}\right\rceil .
$$

In view of Proposition 3.4 there holds

$$
p_{\left|A_{s}\right|}=4 \alpha_{\left|A_{s}\right|}^{2}-4\left\lfloor\alpha_{\left|A_{s}\right|}^{2}-\alpha_{\left|A_{s}\right|}\right\rfloor-6
$$




$$
\begin{aligned}
& \leq 4\left(\frac{3}{2} s \sqrt{3+\frac{1}{s^{2}}}\right)-2 \\
& =6 \sqrt{3} s+\frac{6}{s\left(\sqrt{3}+\sqrt{3+\frac{1}{s^{2}}}\right)}-2 \\
& <6 \sqrt{3} s+\frac{\sqrt{3}}{s}-2<12 s-6
\end{aligned}
$$

for $s \geq 3$.

By combining (28) and (29) we obtain claim (27) for $s$ odd. The result for $s$ even, $s \geq 4$ follows via analogous computations. In view of Proposition 3.4 and (27) armchair hexagons are not extremizers of the isoperimetric inequality, and hence are not ground states.

\section{The Radius of the $n$-WulfF Shape}

For simplicity in what follows we will refer to the elements of $\mathcal{H}_{s}^{Z}$ as hexagons of side $s$, omitting the word zigzag. We first introduce the notion of maximal hexagon associated to a ground state.

Let $G_{n}$ be a ground state in the hexagonal lattice $\mathcal{L}$. Let

$$
r_{G_{n}}:=\max \left\{s \in \mathbb{N}: \text { there exists a point } q \in \mathcal{L} \text { such that } D_{6 s^{2}}+q \subseteq G_{n}\right\} .
$$

For every $q \in \mathcal{L}$ such that $D_{6 r_{G_{n}}^{2}}+q \subset G_{n}$, we will refer to the set

$$
H_{G_{n}}:=D_{6 r_{G_{n}}^{2}}+q
$$

as a maximal hexagon associated to $G_{n}$. We recall that

$$
G_{n}=\bigcup_{k=0}^{2} G_{n}^{k},
$$

where

$$
G_{n}^{k}:=\left\{x \in G_{n}: \Delta_{G_{n}}(x)=k\right\} .
$$

Let us preliminary check that maximal hexagons are non-degenerate for $n>6$. We recall that the $n$-Wulff shape $W_{n}$ is the zigzag hexagon centered in the origin with side $r_{n}$ (see (5)), i.e.,

$$
W_{n}:=D_{6 r_{n}^{2}}
$$

Proposition 5.1. The radius $r_{n}$ of the $n$-Wulff shape $W_{n}$ (see (5) and Theorem 1.2) with $n>6$, satisfies $r_{n} \geq 1$.

Proof. Let $n \in \mathbb{N}$ be such that there exists a ground state $G_{n}$ with $r_{G_{n}}=0$. Then $G_{n}$ does not contain any set of the form $D_{6}+q$ with $q \in \mathcal{L}$, that is, for every $x \in G_{n}$, there holds (see (21))

$$
x \notin G_{n}^{2} .
$$

By (20) and (21), property (30) is equivalent to the claim that every element of $G_{n} \backslash G_{n}^{0}$ contributes to the overall perimeter of $G_{n}$ and the contribution of each element is exactly 2. Since we are assuming that $G_{n}$ is connected (i.e. $\left|G_{n}^{0}\right|=1$ ), this implies that

$$
P\left(G_{n}\right) \geq 2(n-1)
$$


By Proposition 3.4 it follows that

$$
4\left(\alpha_{n}\right)^{2}-4\left\lfloor\left(\alpha_{n}\right)^{2}-\alpha_{n}\right\rfloor-6=P\left(D_{n}\right)=p_{n} \geq 2(n-1),
$$

which in turn implies

$$
n-1 \geq\left\lfloor\left(\alpha_{n}\right)^{2}-\alpha_{n}\right\rfloor \geq\left(\alpha_{n}\right)^{2}-\alpha_{n},
$$

and finally yields $n-\sqrt{6 n} \leq 0$, that is $0 \leq n \leq 6$.

Fix $n \in \mathbb{N}$ and let $G_{n}$ be a connected ground state. We aim at proving an estimate from below on the radius $r_{G_{n}}$ of $H_{G_{n}}$ in terms of the number $n$ of atoms. We first introduce some definitions.

Definition 5.2 (zigzag path). Let $\ell$ be a line orthogonal to one of the three diameters of a minimal simple cycle of the lattice and intersecting $\mathcal{L}$. The zigzag path identified by $\ell$ is the union of points $p \in \mathcal{L}$ such that either $p \in \ell$ or there exists a minimal simple cycle $H$ such that $p$ belongs to a minimal simple cycle $H$ of $\mathcal{L}$, and the two atoms in $H$ bonded to $P$ are in $\ell$. Note that each point of a zigzag path divides it into two half zigzag paths (see Figure 5).

Let $P_{1}, \ldots, P_{4} \in \mathcal{L} \cap \partial F\left(C_{n}\right)$ be such that $P_{1}$ is bonded to $P_{2}$, and for $i=2,3$ the point $P_{i}$ is bonded both to $P_{i-1}$ and $P_{i+1}$. If there exists a unique zigzag path passing through all the points $P_{1}, \ldots, P_{4}$ we will say that this zigzag path is a side of $C_{n}$. If two different (non parallel) zigzag path intersect in the unitary segment joining $P_{2}$ and $P_{3}$ we will refer to this segment as a corner edge of $C_{n}$ (see Figure 4).

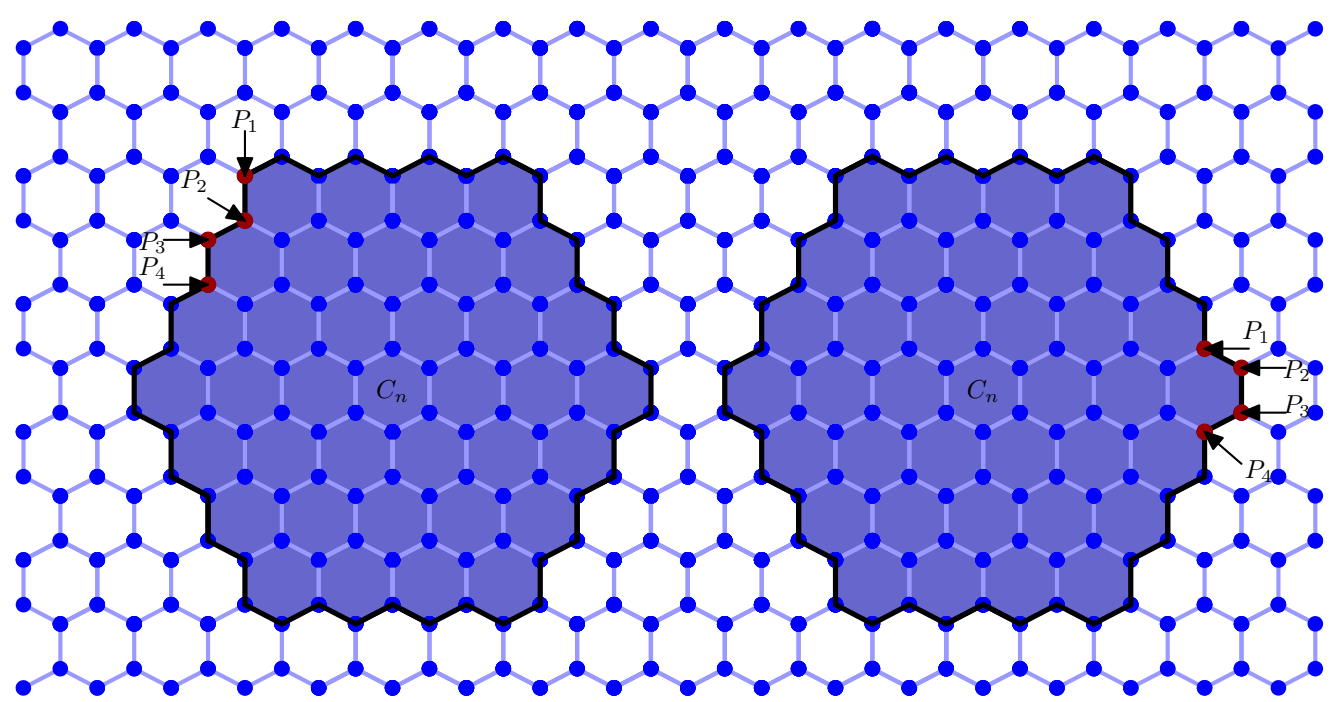

Figure 4 . On the left, the points $P_{1}, \ldots, P_{4}$ belong to a side of $C_{n}$, on the right the segment joining $P_{2}$ and $P_{3}$ is a corner edge of $C_{n}$.

We will say that $C_{n}$ has an angle $\alpha$ in a corner edge $v$ (or in a point $P$ ) if the two lines $\ell_{\alpha}^{1}$ and $\ell_{\alpha}^{2}$, identifying the sides of $C_{n}$ and passing through $v$ (respectively, 
$P$ ), intersect forming an angle of width $\alpha$. The choice of $\alpha$ or $2 \pi-\alpha$ will be clear from the context. Alternatively, we will say that the zigzag paths associated to $\ell_{\alpha}^{1}$ and $\ell_{\alpha}^{2}$ form an angle $\alpha$ with (or in) $v$ (respectively, $P$ ).

Finally, if $S \subset \mathcal{L}$ is such that $\partial(F(S)) \cap \mathcal{L}$ (see Section 2) is the union of two zigzag paths forming an angle $\alpha$, we will call $S$ an angular sector of width $\alpha$, see Figure 5.

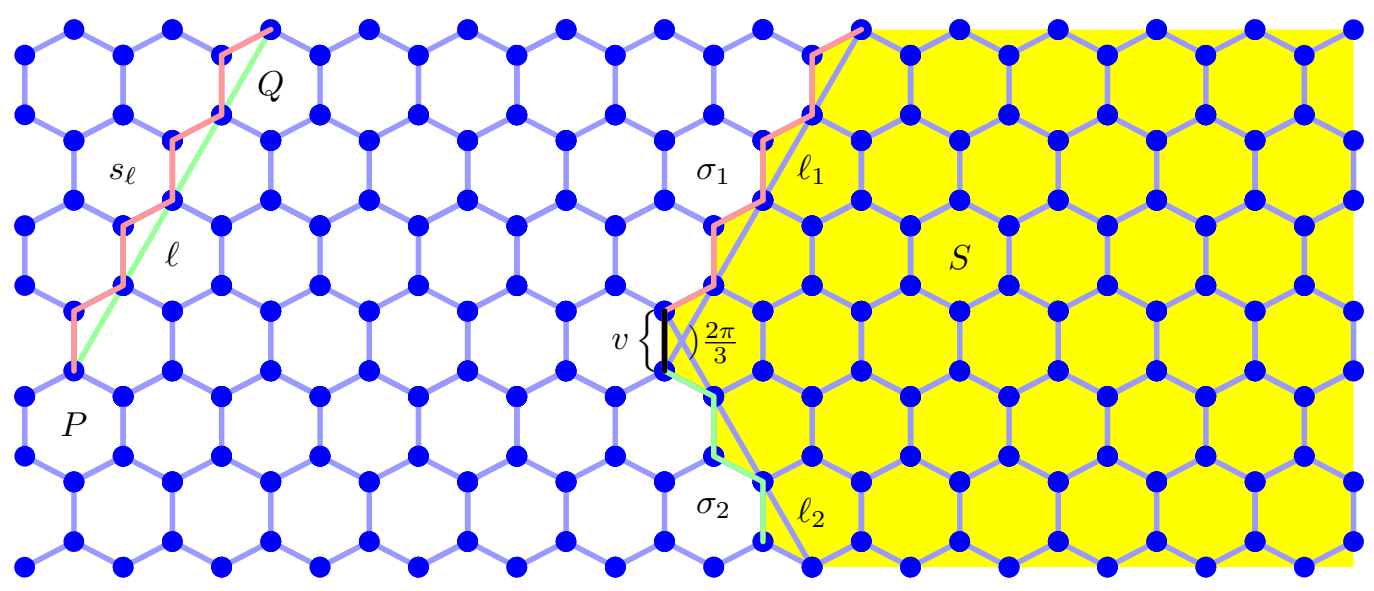

Figure 5 . On the left, the zigzag path $s_{\ell}$ originated by the line $\ell$. On the right, the two zigzag paths $\sigma_{1}$ and $\sigma_{2}$ intersect in the corner edge $v$, forming an angle $2 \pi / 3$. The associated angular sector $S$ is marked in yellow.

By Proposition 5.1 we can assume that $r_{G_{n}} \geq 1$. Let $v_{0}, \ldots, v_{5}$ be the corner edges of $H_{G_{n}}$, where $v_{0}$ is assumed to be lying on the $x$-axis (without loss of generality), and $v_{1}, \ldots, v_{5}$ are numbered counterclockwise starting from $v_{0}$. For $k=0, \ldots, 4$, let $s_{k}$ be the zigzag path joining $v_{k}$ and $v_{k+1}$, and $s_{5}$ be the zigzag path joining $v_{5}$ and $v_{0}$. Let $l_{k}$ be the line identifying the path $s_{k}$, and denote by $\nu_{k}$ the unit normal to $l_{k}$ pointing toward the exterior of $H_{G_{n}}$. We define

$$
\lambda_{k}:=\max \left\{j \in \mathbb{N}: s_{k}^{j} \cap G_{n} \neq 0\right\},
$$

where

$$
s_{k}^{j}:=s_{k}+j \nu_{k}
$$

for $j \in \mathbb{N}$. Let also $\pi_{k}$ be the subset of $\mathcal{L}$ such that $\partial F\left(\pi_{k}\right)=s_{k}$ and $\stackrel{\circ}{F}\left(\pi_{k}\right) \cap H_{G_{n}}=$ $\emptyset$.

We show now that ground states satisfy a connectedness property with respect to zigzag paths.

Definition 5.3 (hex-connectedness). Let $\mathcal{S}$ be a subset of $\mathcal{L}$ and let $P \in \mathcal{L}$. We say that $P$ disconnects a zigzag path in $\mathcal{S}$ if $P \notin \mathcal{S}$ and there exist $P_{a}, P_{b} \in \mathcal{S}$ such that $P_{a}$ and $P_{b}$ are joined by a zigzag path passing through $P$.

Let $\mathcal{S}$ be a subset of $\mathcal{L}$. We say that $\mathcal{S}$ is hex-connected if every $P \in \mathcal{L}$ disconnects at most one zigzag path in $\mathcal{S}$. 
Notice that from every point $P \in \mathcal{L}$ there are exactly three nonparallel lines which depart from $P$ and identify a zigzag path (see Definition 5.2).

Proposition 5.4. Ground states are hex-connected.

Proof. For the sake of contradiction assume that there exists a ground state $G_{n}$ which is not hex-connected. Then there exists a point $P \in \mathcal{L}$ which disconnects two zigzag paths in $G_{n}$. In particular, there exists a line $\ell_{0}$ orthogonal to one of the diameters of a minimal simple cycle of the lattice, and intersecting $\mathcal{L}$, such that the two half zigzag paths starting from $P$ and identified by $\ell_{0}$ are both intersecting $G_{n}$. Let $\ell_{1}, \ldots, \ell_{m}$ be the lines parallel to $\ell_{0}$, intersecting $G_{n}$, and such that for every $i=1, \ldots, m$, the distance between $\ell_{i}$ and $\ell_{0}$ is given by $3 n_{i} / 2$, where $n_{i} \in \mathbb{N}$. For $i=0, \ldots, m$, let $c_{i}$ be the number of points of $G_{n}$ contained in the zigzag path identified by $\ell_{i}$.

We first rearrange the set $\left\{c_{i}\right\}$ in a decreasing order, constructing another set $\left\{d_{i}\right\}$ with the property that $d_{0} \geq d_{1} \geq \cdots \geq d_{m}$. Then, we separate the elements of $\left\{d_{i}\right\}$ having odd indexes from those having even indexes and we consider a new family $\left\{f_{i}\right\}$ obtained by first taking into account the elements of $\left\{d_{i}\right\}$ with even indexes, in decreasing order with respect to their indexes, and then the elements of $\left\{d_{i}\right\}$ having odd indexes, with increasing order with respect to their indexes. In particular we define

$$
\begin{aligned}
& f_{i}:=\left\{\begin{array}{ll}
d_{m-2 i} & i=0, \ldots, \frac{m}{2} \\
d_{2 i-m-1} & i=\frac{m}{2}+1, \ldots, m
\end{array} \quad \text { if } m\right. \text { is even, and } \\
& f_{i}:=\left\{\begin{array}{ll}
d_{m-1-2 i} & i=0, \ldots, \frac{(m-1)}{2} \\
d_{2 i-m} & i=\frac{(m+1)}{2}, \ldots, m
\end{array} \quad \text { if } m \text { is odd } .\right.
\end{aligned}
$$

The set $\left\{f_{i}\right\}$ constructed above has the property that its central elements have the maximum value, and the values of the elements decrease in an alternated way by moving from the center of $\left\{f_{i}\right\}$ toward $i=0$ and $i=m$. Let $\bar{i}$ and $\bar{i}+1$ be the indexes corresponding to the two central elements of the set $\left\{f_{i}\right\}$, if $m$ is odd, and to the central element of $\left\{f_{i}\right\}$ and the maximum between its two neighbors, if $m$ is even. As an example, if we start with a set $\left\{c_{i}\right\}=\{3,4,7,8,2,2,8\}$, the family $\left\{d_{i}\right\}$ is given by $\{8,8,7,4,3,2,2\}$ and the set $\left\{f_{i}\right\}$ by $\{2,3,7,8,8,4,2\}$. Here $\bar{i}=4$.

Fix two points $P_{i}, P_{i+1} \in \mathcal{L}_{h}$ such that the segment $P_{i} P_{i+1}$ has length one and is orthogonal to $\ell_{0}$. Let $\sigma_{1}$ and $\sigma_{2}$ be two half-zigzag paths, starting from $P_{i}$ and $P_{i+1}$, respectively, forming an angle $2 \pi / 3$ with $P_{i} P_{i+1}$, and such that there exists a convex region $S$ of the plane whose boundary is given by $\sigma_{1}, \sigma_{2}$, and $P_{i} P_{i+1}$.

Consider the points $P_{0}, \ldots, P_{\bar{i}-1} \in \sigma_{1}$, defined as

$$
\left|P_{i}-P_{j}\right|=(\bar{i}-j) \sqrt{3}, \quad j=0, \ldots, \bar{i}-1 .
$$

Analogously, consider the points $P_{i+2}, \ldots, P_{m} \in \sigma_{2}$, satisfying

$$
\left|P_{i+1}-P_{j}\right|=(j-\bar{i}) \sqrt{3}, \quad j=\bar{i}+2, \ldots, m \text {. }
$$

For $j=0, \ldots, m$, let $\tilde{\ell}_{j}$ be the line parallel to $\ell_{0}$ and passing through $P_{j}$. To construct the set $\tilde{G}_{n}$ we consider $f_{j}$ consecutive points in $S$ on the zigzag path 
identified by each line $\tilde{\ell}_{j}$, starting from $P_{j}$ (see figure 5 ). The set $\tilde{G}_{n}$ is clearly hexconnected, the number of bonds in the zigzag paths identified by the lines parallel to $\ell_{0}$ has increased, and the number of bonds between parallel zigzag paths has not decreased. Hence

$$
E\left(\tilde{G}_{n}\right)<E\left(G_{n}\right)
$$

providing a contradiction to the optimality of the ground state $G_{n}$.

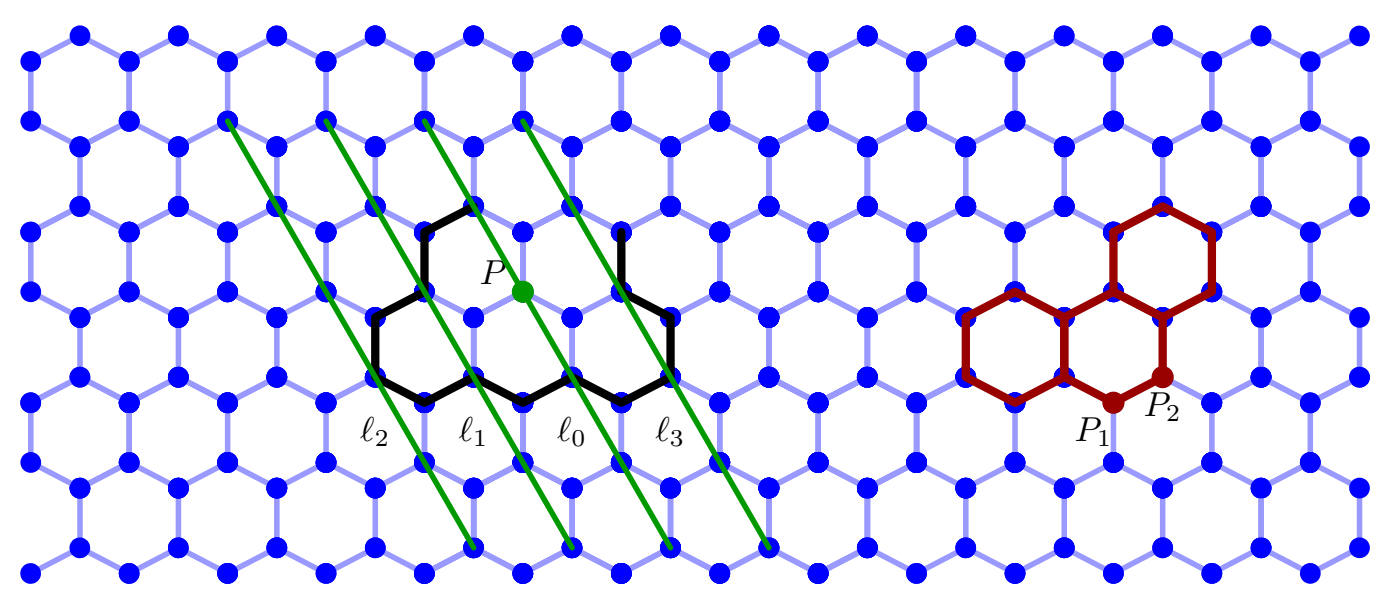

Figure 6. A configuration $C_{n}$ before (in black) and after (in red) the rearrangement described in Proposition 5.4

As a corollary of Proposition 5.4 it follows that ground states have no vacancies.

Proposition 5.5. Let $G_{n}$ be a ground state. Then $F\left(G_{n}\right)$ is simply connected.

Proof. By contradiction, if $F\left(G_{n}\right)$ is not simply connected then there exists a point in $\mathcal{L}$ that disconnects three zigzag paths in $G_{n}$. Therefore $G_{n}$ is not hex-connected.

We conclude this overview on connectedness properties of ground states with the following proposition.

Proposition 5.6. Ground states are connected.

We omit the proof of this result as it follows by adapting the proof of Proposition 5.4.

In view of Propositions 5.4-5.6, the quantity $\lambda_{k}$ defined in (31) provides the number of nonempty parallel zigzag paths of atoms in $G_{n} \cap \pi_{k}$. By the definition of $\tau$, each partially full level of atoms around $H_{G_{n}}$ is characterized by the fact that the difference between the number of points on the level having weight one and those having weight two is strictly positive. To be precise,

$$
\sum_{k=0}^{5} \lambda_{k} \leq\left|G_{n}^{1} \backslash H_{G_{n}}\right|-\left|G_{n}^{2} \backslash H_{G_{n}}\right| .
$$


On the other hand, by (20) and (21),

$$
\left|G_{n}^{1} \backslash H_{G_{n}}\right|-\left|G_{n}^{2} \backslash H_{G_{n}}\right|=\frac{P\left(G_{n}\right)-P\left(H_{G_{n}}\right)}{2}=\frac{p_{n}}{2}-6 r_{G_{n}}+3,
$$

thus yielding

$$
\sum_{k=0}^{5} \lambda_{k} \leq \frac{p_{n}}{2}-6 r_{G_{n}}+3 .
$$

Given a ground state $G_{n}$ and its maximal hexagon $H_{G_{n}}:=D_{6 r_{G_{n}}^{2}}+q$, denote by $H_{G_{n}}^{+}$and $H_{G_{n}}^{++}$the sets $H_{G_{n}}^{+}:=D_{6\left(r_{G_{n}}+1\right)^{2}}+q$, and $H_{G_{n}}^{++}:=D_{6\left(r_{G_{n}}+2\right)^{2}}+q$, respectively. Denote by $v_{i}^{\prime}$ and $v_{i}^{\prime \prime}, i=0, \ldots, 5$ the corner edges of $H_{G_{n}}^{+}$and $H_{G_{n}}^{++}$, respectively, with the convention that both $v_{i}^{\prime}$ and $v_{i}^{\prime \prime}$ are parallel to $v_{i}$. For $i=0, \ldots, 5$, let $V_{i}^{1}$ and $V_{i}^{2}$ be the two extrema of $v_{i}$, numbered counterclockwise. Let $\left(V_{i}^{\prime}\right)^{1},\left(V_{i}^{\prime}\right)^{2},\left(V_{i}^{\prime \prime}\right)^{1},\left(V_{i}^{\prime \prime}\right)^{2}, s_{i}^{\prime}$, and $s_{i}^{\prime \prime}$ be defined accordingly.

In the remaining part of this subsection we provide a characterization of the geometry of $G_{n} \backslash H_{G_{n}}^{+}$, by subdividing this set into good polygons $P_{k}$ and bad polygons $T_{k}$, and by showing that the cardinality of $G_{n} \backslash H_{G_{n}}^{+}$is, roughly speaking, the same as the one of the union of good polygons.

We first prove that, by the optimality of $H_{G_{n}}$, there exists a corner edge of $H_{G_{n}}^{++}$ which does not intersect $G_{n}$.

Proposition 5.7. Let $G_{n}$ be a ground state, and $H_{G_{n}}$ be its maximal hexagon. Then there exists a corner edge $v_{j}^{\prime \prime}$ of $H_{G_{n}}^{++}, j=0, \cdots, 5$, which does not intersect $G_{n}$.

Proof. By the maximality of $H_{G_{n}}$, there exists a point $P \in \partial F\left(H_{G_{n}}^{+}\right)$such that $P \notin G_{n}$.

If $P$ does not disconnect $s_{i}^{\prime}$ then either $v_{i}^{\prime}$ or $v_{i+1}^{\prime}$ do not intersect $G_{n}$. By the hex-connectedness of $G_{n}$ (see Proposition 5.4) then also the corresponding corner edge of $H_{G_{n}}^{\prime \prime}$ does not intersect $G_{n}$, and we obtain the thesis.

Assume now that $P$ disconnects $s_{i}^{\prime}$. Since $G_{n}$ is hex-connected, the point $P$ does not disconnect any other zigzag path. Therefore there exists an angular sector $S$ centered in $P$ and of width $\pi / 3$ such that

$$
S \cap G_{n}=\emptyset .
$$

Assume by contradiction that all corner edges of $H_{G_{n}}^{++}$intersect $G_{n}$. In view of (33), the set $\left(G_{n} \backslash H_{G_{n}}\right) \cap \pi_{i}$ is subdivided into two components. Denoting them by $\Gamma_{1}$ and $\Gamma_{2}$, we have that $\Gamma_{j} \cap s_{i}^{1} \neq \emptyset$ and $\Gamma_{j} \cap s_{i}^{2} \neq \emptyset$, for $j=1,2$. Without loss of generality we can assume that $\Gamma_{1} \cap s_{i}^{\lambda_{i}} \neq \emptyset$. Consider now the set $M:=\Gamma_{1} \cap\left(s_{i}^{\lambda_{i}} \cup s_{i}^{\lambda_{i}-1}\right)$. We claim that we can construct a new set $\tilde{G}_{n}$, by rearranging the atoms of $M$ and by leaving the other elements of $G_{n}$ fixed, such that

$$
E\left(\tilde{G}_{n}\right)<E\left(G_{n}\right) .
$$

There are three possible scenarios.

Case 1: $\Gamma_{1}$ contains at least two points $P_{a}$ and $P_{b}$ with the property that for each of them there is no minimal cycle passing through it and entirely contained in 
$G_{n}$. We proceed by moving the two points to $s_{i}^{1} \cap\left(\mathcal{L} \backslash G_{n}\right)$ in such a way that $P_{a}$ is bonded to $\Gamma_{2}$. If possible, we move also $P_{b}$ to $s_{i}^{1} \cap\left(\mathcal{L} \backslash G_{n}\right)$ so that $P_{a}$ and $P_{b}$ are bonded. If this is not possible because $s_{i} \cap\left(\mathcal{L} \backslash G_{n}\right)$ contains only one element, then we already created an extra bond. With this procedure we loose two bonds when removing $P_{a}$ and $P_{b}$ from $\Gamma_{1}$, but we gain at least three bonds when we attach them to $\Gamma_{2}$, therefore the total energy strictly decreases.

Case 2: in $\Gamma_{1}$ there exists exactly one point $P_{a}$ with the property that there is no minimal cycle containing it and entirely contained in $G_{n}$. We argue moving this single point to $s_{i}^{1} \cap\left(\mathcal{L} \backslash G_{n}\right)$ in such a way that $P_{a}$ is bonded to $\Gamma_{2}$. Afterward, we move iteratively all the (remaining) points in $s_{i}^{\lambda_{i}} \cap \Gamma_{1}$ to $s_{i}^{1} \cap\left(\mathcal{L} \backslash G_{n}\right)$ (in the same way as described in Case 1 for $P_{b}$ ). If after moving $P_{a}$ there are no remaining points in $s_{i}^{\lambda_{i}} \cap \Gamma_{1}$, we apply the same rearrangement to $s_{i}^{\lambda_{i}-1} \cap \Gamma_{1}$ (note that $\lambda_{i} \geq 2$ because all corner edges of $H_{G_{n}}^{++}$intersect $G_{n}$ ). As a result of the procedure described above, the energy is strictly decreased.If at any moment during the process of attaching points to $\Gamma_{2}$ we create a bond between $\Gamma_{1}$ and $\Gamma_{2}$, we stop the rearrangement as the number of bonds has strictly increased.

Case 3: every point of $\Gamma_{1}$ belongs to a minimal cycle entirely contained in $G_{n}$. In this case we first move all points in $s_{i}^{\lambda_{i}} \cap \Gamma_{1}$ but one, in the same way as described in Cases 1 and 2. As a result of this procedure, either we already created an extra bond (and hence there is nothing left to prove) or we are now in the same situation described in Case 2. The thesis follows then arguing exactly as in Case 2.

We proceed by showing that for every hexagon of side $r_{H}+2$ there exists an angular sector of width $\pi / 3$, and centered in one of its corner edges, which does not intersect $G_{n}$.

Proposition 5.8. Let $G_{n}$ be a ground state, and $H_{G_{n}}$ be its maximal hexagon. Then

(i) There exists a corner edge $v_{i}^{\prime \prime}$ of $H_{G_{n}}^{++}, i=0, \ldots, 5$, and an angular sector $S$ of width $\pi / 3$, centered in $\left(V_{i}^{\prime \prime}\right)^{1}$ or $\left(V_{i}^{\prime \prime}\right)^{2}$, and such that $S \cap G_{n}=\emptyset$.

(ii) Every hexagon in $\mathcal{L}$ with side $r_{G_{n}}+2$ has a corner edge and a corresponding angular sector of width $\pi / 3$ which do not intersect $G_{n}$.

Proof. By Proposition 5.7 we can assume that $v_{0}^{\prime \prime}$ does not intersect $G_{n}$. Assume first that both $\left(V_{0}^{\prime \prime}\right)^{1}$ and $\left(V_{0}^{\prime \prime}\right)^{2}$ do not disconnect any zigzag path. Consider the two half zigzag paths in which $v_{0}^{\prime \prime}$ divides $s_{0}^{\prime \prime}$. Then at least one of them does not intersect $G_{n}$. Analogously, at least one of the two half zigzag paths in which $v_{0}^{\prime}$ divides $s_{5}^{\prime}$ does not intersect $G_{n}$. Finally, the two half zigzag paths, departing from $\left(V_{0}^{\prime}\right)^{1}$ and $\left(V_{0}^{\prime}\right)^{2}$, not parallel to $s_{0}$ and $s_{5}$, and in the opposite direction with respect to the center of $H_{G_{n}}$, do not intersect $G_{n}$. According to the geometric position of the four half-zigzag paths identified beforehand, and using again the hex-connectedness of $G_{n}$ we obtain (i), the sector $S$ being of width $2 \pi / 3$. The case in which at least one between $\left(V_{0}^{\prime \prime}\right)^{1}$ and $\left(V_{0}^{\prime \prime}\right)^{2}$ disconnects one zigzag path (see Proposition 5.4) follows accordingly, yielding a sector $S$ of width $\pi / 3$. The proof of (ii) is an adaptation of the proof of (i). 
Without loss of generality, in view of Proposition 5.8 we can assume that $v_{0}^{\prime \prime} \nsubseteq$ $G_{n}$. For $k=2,3,4,5$, let $\pi_{k}^{\prime}$ be the subset of $\mathcal{L}$ such that

$$
\left\{\begin{array}{l}
\pi_{k}^{\prime}=F\left(\pi_{k}^{\prime}\right) \cap \mathcal{L} \\
\partial F\left(\pi_{k}^{\prime}\right) \cap \mathcal{L}=s_{k}^{\lambda_{k}} \\
H_{G_{n}} \subset \pi_{k}^{\prime}
\end{array}\right.
$$

Consider the set $R:=\left(\cap_{k=0}^{5} \pi_{k}^{\prime}\right) \backslash H_{G_{n}}^{+}$.

By construction, $G_{n} \subset H_{G_{n}}^{+} \cup R$, and for every $x \in R$ and $k=0, \ldots, 5$ there exists

$$
j_{k} \in\left[-\lambda_{\left(\frac{k+3}{6}-\left\lfloor\frac{k+3}{6}\right\rfloor\right)}-2 r_{G_{n}}-2, \lambda_{k}\right]
$$

such that $x \in s_{k}^{j_{k}}$. In particular, every $x \in R$ is uniquely determined by a pair of indexes $\left(j_{k}, j_{k^{\prime}}\right)$, with $k^{\prime} \neq k+3$ in $\mathbb{Z}_{6}$.

We subdivide the region $R$ into disjoint polygons as

$$
R=\left(\cup_{j=0}^{5} P_{j}\right) \cup\left(\cup_{j=0}^{5} T_{j}\right) .
$$

For $a \in\left[-2\left(r_{G_{n}}+1\right), 2\left(r_{G_{n}}+1\right)\right]$, denote by $P_{k}^{1}(a)$ the subset of $\mathcal{L}$ enclosed by $s_{k}^{2}$, $s_{k}^{a}, s_{k+1}^{1}, s_{k+1}^{-r_{G_{n}}}$; and by $P_{k}^{2}(a)$ the set delimited by $s_{k}^{a}, s_{k}^{\lambda_{k}}, s_{k-1}^{\lambda_{k-1}-r_{G_{n}}}, s_{k-1}^{\lambda_{k-1}}$. For $k=0, \ldots, 5$, the sets $P_{k}$ in (35) are defined as follows:

$$
P_{k}:= \begin{cases}P_{k}^{1}\left(\lambda_{k}\right) \cap R & \text { if } \lambda_{k} \leq \lambda_{k-1}+1, \\ \left(P_{k}^{1}\left(\lambda_{k}-\lambda_{k-1}+1\right) \cap R\right) \cup P_{k}^{2}\left(\lambda_{k}-\lambda_{k-1}+1\right) & \text { if } \lambda_{k}>\lambda_{k-1}+1,\end{cases}
$$

with the convention that $\lambda_{-1}:=\lambda_{5}$. Note that $\left|P_{k}\right|=2\left(r_{G_{n}}+1\right)\left(\lambda_{k}-1\right)$ for every $k=0, \ldots, 5$.

The sets $T_{k}$ are given, roughly speaking, by the "portions of $\mathcal{L}$ " between $P_{k-1}$ and $P_{k}$. To be precise,

$$
\begin{aligned}
T_{k}:=\left\{x \in R: x \in s_{k-1}^{j_{k-1}} \cap s_{k}^{j_{k}}, \text { with } 2 \leq j_{k-1} \leq \lambda_{k-1}, 2 \leq j_{k} \leq \lambda_{k}, j_{k-1} \geq j_{k}\right. \\
\text { and, if } \left.\lambda_{k-1}>\lambda_{k-2}+1, j_{k-1} \leq j_{k}+\lambda_{k-1}-\lambda_{k-2}\right\}
\end{aligned}
$$

see Figure 7.

We have that

$$
n \leq\left|H_{G_{n}}^{+}\right|+|R|-\left|R \backslash G_{n}\right|
$$

where $\left|H_{G_{n}}^{+}\right|=6\left(r_{G_{n}}+1\right)^{2}$. We observe that

$$
|R|=\sum_{j=0}^{5}\left|P_{j}\right|+\sum_{j=0}^{5}\left|T_{j}\right|=2\left(r_{G_{n}}+1\right) \sum_{j=0}^{5}\left(\lambda_{j}-1\right)+\sum_{j=0}^{5}\left|T_{j}\right|-1 .
$$

We proceed now in counting the points in $R$ which do not belong to the ground state $G_{n}$. In particular, we prove a lower bound for such number in terms of the cardinality of

$$
\mathcal{H}:=\left\{H \subset \mathcal{L} \cap\left(H_{G_{n}}^{+} \cup R\right): H \text { is a hexagon of side } r_{G_{n}}+2\right\} .
$$

\section{Proposition 5.9.}

$$
\left|R \backslash G_{n}\right| \geq 2|\mathcal{H}|
$$




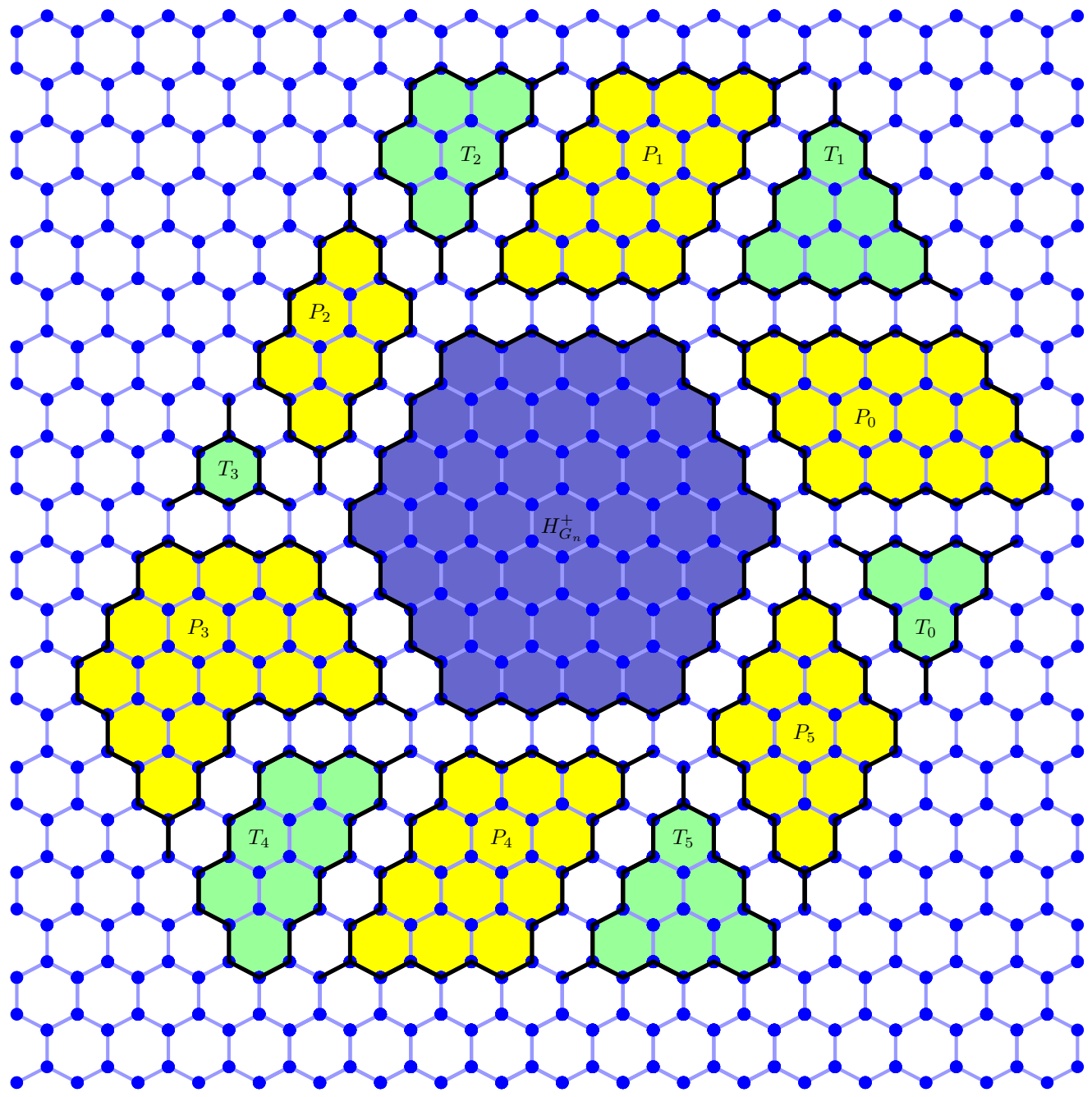

Figure 7 . The structure of a ground state $G_{n}$. In the figure above, $r_{G_{n}}=3, \lambda_{0}=6, \lambda_{1}=6, \lambda_{2}=4, \lambda_{3}=7, \lambda_{4}=6$, and $\lambda_{5}=5$. The blue shape outlined in black, the yellow shapes outlined in black, and the green shapes outlined in black correspond to the closed subsets of the plane associated to $H_{G_{n}}^{+}$, to the parallelograms $P_{j}$, and to the triangles $T_{j}$, respectively.

Proof. Set $M:=|\mathcal{H}|$. We show by induction on $m=1, \ldots, M$ that for every family $\mathcal{H}_{m} \subset \mathcal{H}$ with $\left|\mathcal{H}_{m}\right|=m$, there exists a collection of pairs of bonded atoms $V_{\mathcal{H}_{m}} \subset$ $R \backslash G_{n}$ with $\left|V_{\mathcal{H}_{m}}\right|=m$ satisfying the following property: identifying each segment with its extrema, the correspondence associating to each pair $\left(\nu_{1}, \nu_{2}\right) \in V_{\mathcal{H}_{m}}$ a hexagon $H \in \mathcal{H}_{m}$ having a corner edge in $\left(\nu_{1}, \nu_{2}\right)$ is a bijection. 
We remark that the thesis will follow once we prove the assertion for $m=M$. For $m=1$ the claim holds by Proposition 5.8. Assume now that the claim is satisfied for $m=\bar{m}$. Consider a family $\mathcal{H}_{\bar{m}+1}=\left\{H_{1}, \ldots, H_{\bar{m}+1}\right\} \subset \mathcal{H}$, and the polygon $\mathcal{P}_{\bar{m}+1}:=\cup_{i=1}^{\bar{m}+1} H_{i} \subset R$. We subdivide the remaining part of the proof into 4 steps.

Step 1: there exists a corner edge $\left(\tilde{\nu}_{1}, \tilde{\nu}_{2}\right)$ of $\mathcal{P}_{\bar{m}+1}$ such that $\tilde{\nu}_{i} \in \mathcal{P}_{\bar{m}+1} \backslash G_{n}$ for $i=1,2$.

Indeed, assume by contradiction that every corner edge of $\mathcal{P}_{\bar{m}+1}$ belongs to $G_{n}$. Then every corner edge of $H_{\bar{m}+1}$ in $\stackrel{\circ}{F}\left(\mathcal{P}_{\bar{m}+1}\right)$ would belong to $G_{n}$ by Proposition 5.5 . Thus all corner edges of $H_{\bar{m}+1}$ would belong to $G_{n}$, contradicting Proposition 5.7.

Step 2: by Proposition 5.8 (ii) there exists an angular sector $S$ of width at least $\pi / 3$, centered in $\tilde{\nu}_{1}, \tilde{\nu}_{2}$, or in $\left(\tilde{\nu}_{1}, \tilde{\nu}_{2}\right)$, and such that $\bar{S} \cap G_{n}=\emptyset$. Denote by $\sigma_{1}$ and $\sigma_{2}$ the two zigzag paths forming its boundary.

Step 3: We claim that there exists a corner edge $\left(\nu_{1}, \nu_{2}\right)$ of $\mathcal{P}_{\bar{m}+1}$ such that $\left(\nu_{1}, \nu_{2}\right) \subset \mathcal{P}_{\bar{m}+1} \backslash G_{n}$, and $\left(\nu_{1}, \nu_{2}\right)$ is associated to an angle $2 \pi / 3$ of $\mathcal{P}_{\bar{m}+1}$.

Observe that $\mathcal{P}_{\bar{m}+1}$ can have corner edges with angles $2 \pi / 3,4 \pi / 3$, or $5 \pi / 3$. If the corner edge $\left(\tilde{\nu}_{1}, \tilde{\nu}_{2}\right)$ found in Step 2 is associated to an angle $2 \pi / 3$, there is nothing to prove. If $\left(\tilde{\nu}_{1}, \tilde{\nu}_{2}\right)$ corresponds to an angle $4 \pi / 3$, or $5 \pi / 3$, then there are two possible cases:

Case 1: $F(S) \cap \mathcal{P}_{\bar{m}+1}=\emptyset$. Then, for every $j=1,2$, there exists $\hat{\nu}_{j} \in \sigma_{j}$ such that $\hat{\nu}_{j}$ is one of the two extrema of a corner edge of $\mathcal{P}_{\bar{m}+1}$ entirely contained in $S$, and at least one among the zigzag paths from $\hat{\nu}_{1}$ to $\tilde{\nu}_{1}$ and from $\hat{\nu}_{2}$ to $\tilde{\nu}_{2}$ is contained in $\partial F\left(\mathcal{P}_{\bar{m}+1}\right)$. In addition, the corner edge associated to such $\tilde{\nu}_{j}$ does not intersect $G_{n}$ (because it is a subset of $S$ ), and is associated to an angle $2 \pi / 3$ (since $\left.F(S) \cap \mathcal{P}_{\bar{m}+1}=\emptyset\right)$. The proof follows by considering the corner edge associated to $\hat{\nu}_{j}$.

Case 2: $F(S) \cap \mathcal{P}_{\bar{m}+1} \neq \emptyset$. Let $\ell_{1}$ and $\ell_{2}$ be the lines generating $\sigma_{1}$ and $\sigma_{2}$, and let $n_{1}$ and $n_{2}$ be the unit normal vectors to $\ell_{1}$ and $\ell_{2}$, respectively, pointing outside $S$. Define

$$
\sigma_{1}^{k}:=\sigma_{1}-\frac{3}{2} k n_{1} \quad \text { and } \quad \sigma_{2}^{k}:=\sigma_{2}-\frac{3}{2} k n_{2} .
$$

for $k \in \mathbb{N}$. Since $\mathcal{P}_{\bar{m}+1} \cap S$ is bounded, we can find

$$
k_{1}:=\max \left\{k \in \mathbb{N}: \sigma_{1}^{k} \cap \mathcal{P}_{\bar{m}+1} \cap S \neq \emptyset\right\}
$$

and

$$
k_{2}:=\max \left\{k \in \mathbb{N}: \sigma_{2}^{k} \cap \mathcal{P}_{\bar{m}+1} \cap S \neq \emptyset\right\} .
$$

For $j=1,2$, the intersection $\sigma_{j}^{k_{j}} \cap \partial F\left(\mathcal{P}_{\bar{m}+1}\right) \cap S$ is either a corner edge of $\mathcal{P}_{\bar{m}+1}$ associated to an angle $2 \pi / 3$, or a zigzag path forming an angle $2 \pi / 3$ with a corner edge of $\mathcal{P}_{\bar{m}+1}$. 
Step 4: Let $\left(\nu_{1}, \nu_{2}\right)$ be the corner edge provided by Step 3. Then, there exists a unique $H_{\bar{j}} \in \mathcal{H}_{\bar{m}+1}$ having a corner edge identified by $\left(\nu_{1}, \nu_{2}\right)$. Thus, by the induction hypothesis on $\left\{H_{1}, \ldots, H_{\bar{m}+1}\right\} \backslash\left\{H_{\bar{j}}\right\}$, there exists a family of corner edges

$$
\left\{\left(\nu_{1}^{j}, \nu_{2}^{j}\right)\right\}_{j=1, \ldots, \bar{m}+1, j \neq \bar{j}} \subset R \backslash G_{n}
$$

such that, for every $j,\left(\nu_{1}^{j}, \nu_{2}^{j}\right)$ is a corner edge of $H_{j}$, and for every $i \neq j\left(\nu_{1}^{j}, \nu_{2}^{j}\right)$ is not a corner edge of $H_{i}$. The thesis follows by setting $\left(\nu_{1}^{\bar{j}}, \nu_{2}^{\bar{j}}\right)=\left(\nu_{1}, \nu_{2}\right)$, and taking $V_{\mathcal{H}_{\bar{m}+1}}=\left\{\left(\nu_{1}^{1}, \nu_{2}^{1}\right), \ldots,\left(\nu_{1}^{\bar{m}+1}, \nu_{2}^{\bar{m}+1}\right)\right\}$.

The next step consists in estimating $|\mathcal{H}|$ from below, in terms of the cardinality of the sets $T_{j}$ and the number of levels $\lambda_{j}$.

\section{Proposition 5.10.}

$$
2|\mathcal{H}| \geq \sum_{j=0}^{5}\left|T_{j}\right|-2 \lambda_{1}-4 \lambda_{2}-4 \lambda_{3}-4 \lambda_{4}-2 \lambda_{5}+18
$$

Proof. For $k=2,3,4,5$, let $U_{k}$ be the region of $\mathcal{L}$ containing $H_{G_{n}}^{+}$and delimited by the zigzag paths $s_{k+1}^{1}, s_{k+2}^{1}$, and $s_{k+3}^{1}$ (with $s_{6}^{1}:=s_{0}^{1}, s_{7}^{1}:=s_{1}^{1}$ and $s_{8}^{1}:=s_{2}^{1}$ ). Let $\mathcal{H}_{k}:=\left\{H \in \mathcal{H}: H \subset U_{k}\right.$ and has a vertex in $\left.T_{k}\right\}, k=2,3,4,5$.

We claim that

$$
\left|\mathcal{H}_{k}\right| \geq \frac{\left|T_{k}\right|-2\left(\lambda_{k}+\lambda_{k-1}-3\right)}{2} .
$$

Indeed, let $(\tilde{x}, \hat{x}) \in T_{k}$ and consider $\left(\tilde{j}_{k}, \tilde{j}_{k-1}, \tilde{j}_{k-2}\right)$ such that $\tilde{x} \in s_{k}^{\tilde{j}_{k}} \cap s_{k-1}^{\tilde{j}_{k-1}} \cap$ $s_{k-2}^{\tilde{j}_{k-2}}$ and $\hat{x} \in s_{k}^{\tilde{j}_{k}} \cap s_{k-1}^{\tilde{j}_{k-1}} \cap s_{k-2}^{\tilde{j}_{k-2}-1}$. We identify $\tilde{x}$ and $\hat{x}$ with the triple of indexes $\left(\tilde{j}_{k}, \tilde{j}_{k-1}, \tilde{j}_{k-2}\right)$ and $\left(\tilde{j}_{k}, \tilde{j}_{k-1}, \tilde{j}_{k-2}-1\right)$, and we write $\tilde{x}=\left(\tilde{j}_{k}, \tilde{j}_{k-1}, \tilde{j}_{k-2}\right)$ and $\hat{x}=$ $\left(\tilde{j}_{k}, \tilde{j}_{k-1}, \tilde{j}_{k-2}-1\right)$. Let $H_{\tilde{x}, \hat{x}}$ be the hexagon with corner edges identified by the pairs $(\tilde{x}, \hat{x})$, and the pairs

$$
\begin{aligned}
w_{1}:= & {\left[\left(\tilde{j}_{k}-\left(r_{G_{n}}+2\right), \tilde{j}_{k-1}, \tilde{j}_{k-2}+\left(r_{G_{n}}+1\right)\right),\left(\tilde{j}_{k}-\left(r_{G_{n}}+1\right), \tilde{j}_{k-1}, \tilde{j}_{k-2}+\left(r_{G_{n}}+1\right)\right)\right] } \\
w_{2}:= & {\left[\left(\tilde{j}_{k}-\left(2 r_{G_{n}}+3\right), \tilde{j}_{k-1}-\left(r_{G_{n}}+2\right), \tilde{j}_{k-2}+\left(r_{G_{n}}+1\right)\right),\right.} \\
& \left.\left(\tilde{j}_{k}-\left(2 r_{G_{n}}+3\right), \tilde{j}_{k-1}-\left(r_{G_{n}}+1\right), \tilde{j}_{k-2}+\left(r_{G_{n}}+1\right)\right)\right], \\
w_{3}:=\left[\left(\tilde{j}_{k}-\left(2 r_{G_{n}}+3\right), \tilde{j}_{k-1}-\left(2 r_{G_{n}}+3\right), \tilde{j}_{k-2}-1\right),\right. & \left.\left(\tilde{j}_{k}-\left(2 r_{G_{n}}+3\right), \tilde{j}_{k-1}-\left(2 r_{G_{n}}+3\right), \tilde{j}_{k-2}\right)\right], \\
w_{4}:= & {\left[\left(\tilde{j}_{k}-\left(r_{G_{n}}+1\right), \tilde{j}_{k-1}-\left(2 r_{G_{n}}+3\right), \tilde{j}_{k-2}-\left(r_{G_{n}}+2\right)\right),\right.} \\
& \left.\left(\tilde{j}_{k}-\left(r_{G_{n}}+2\right), \tilde{j}_{k-1}-\left(2 r_{G_{n}}+3\right), \tilde{j}_{k-2}-\left(r_{G_{n}}+2\right)\right)\right], \\
w_{5}:= & {\left[\left(\tilde{j}_{k}, \tilde{j}_{k-1}-\left(r_{G_{n}}+2\right), \tilde{j}_{k-2}-\left(r_{G_{n}}+2\right)\right),\left(\tilde{j}_{k}, \tilde{j}_{k-1}-\left(r_{G_{n}}+1\right), \tilde{j}_{k-2}-\left(r_{G_{n}}+2\right)\right)\right] . }
\end{aligned}
$$

We observe that $H_{\tilde{x}, \hat{x}}$ is contained in $U_{k}$ and has a corner edge in $T_{k}$ if the following inequalities are satisfied

$$
\begin{aligned}
& \tilde{j}_{k}-\left(2 r_{G_{n}}+3\right) \geq-2 r_{G_{n}}-1, \quad \tilde{j}_{k} \leq \lambda_{k}, \\
& \tilde{j}_{k-1}-\left(2 r_{G_{n}}+3\right) \geq-2 r_{G_{n}}-1, \quad \tilde{j}_{k-1} \leq \lambda_{k-1}, \\
& \tilde{j}_{k-2}-\left(r_{G_{n}}+2\right) \geq-2 r_{G_{n}}-1, \quad \tilde{j}_{k-2}+r_{G_{n}}+1 \leq \lambda_{k-2} .
\end{aligned}
$$


Hence, if $\left(\tilde{j}_{k}, \tilde{j}_{k-1}, \tilde{j}_{k-2}\right)$ is such that

$$
\begin{aligned}
2 & \leq \tilde{j}_{k} \leq \lambda_{k} \\
2 & \leq \tilde{j}_{k-1} \leq \lambda_{k-1} \\
-r_{G_{n}}+1 & \leq \tilde{j}_{k-2} \leq \lambda_{k-2}-r_{G_{n}}-1
\end{aligned}
$$

then $H_{\tilde{x}, \hat{x}} \subset U_{k}$ and has a corner edge in $T_{k}$. By the definition of the sets $T_{k}$ (see (36)), the previous properties are fulfilled by every $x \in T_{k}$, apart from those points belonging to the portion of $\partial F\left(T_{k}\right)$ which is adjacent either to $P_{k-1}$ or to $P_{k}$. Denoting by $\tilde{T}_{k}$ this latter set, claim (37) follows once we observe that

$$
\left|\tilde{T}_{k}\right|=\frac{\left|T_{k}\right|-2\left(\lambda_{k}+\lambda_{k-1}-3\right)}{2} .
$$

Combining Propositions 5.9 and 5.10 we estimate from above and from below the radius $r_{G_{n}}$ of the maximal hexagon $H_{G_{n}}$.

\section{Proposition 5.11.}

$$
\rho_{n} \leq r_{n} \leq R_{n} \leq \rho_{n}+\frac{2}{3} \sqrt{\left[\left(\alpha_{n}\right)^{2}-\left\lfloor\left(\alpha_{n}\right)^{2}-\alpha_{n}\right\rfloor\right]^{2}-\left(\alpha_{n}\right)^{2}+39},
$$

where $R_{n}:=\max \left\{r_{G_{n}}: G_{n}\right.$ is a ground state with $n$ atoms $\}$ and

$$
\rho_{n}:=\frac{\left(\alpha_{n}\right)^{2}}{3}-\frac{\left\lfloor\left(\alpha_{n}\right)^{2}-\alpha_{n}\right\rfloor}{3}-3-\frac{1}{3} \sqrt{\left[\left(\alpha_{n}\right)^{2}-\left\lfloor\left(\alpha_{n}\right)^{2}-\alpha_{n}\right\rfloor\right]^{2}-\left(\alpha_{n}\right)^{2}+39},
$$

with $\alpha_{n}=\sqrt{3 n / 2}$.

Proof. By Propositions 5.9 and 5.10 we have

$$
\left|R \backslash G_{n}\right| \geq \sum_{j=0}^{5}\left|T_{j}\right|-2 \lambda_{1}-4 \lambda_{2}-4 \lambda_{3}-4 \lambda_{4}-2 \lambda_{5}+18
$$

Therefore, by (32) we obtain

$$
\begin{aligned}
n & \leq\left|H_{G_{n}}^{+}\right|+|R|-\left|R \backslash G_{n}\right| \\
& \leq 6\left(r_{G_{n}}+1\right)^{2}+\sum_{j=0}^{5}\left|P_{j}\right|+\sum_{j=0}^{5}\left|T_{j}\right|-\sum_{j=0}^{5}\left|T_{j}\right|+2 \lambda_{1}+4 \lambda_{2}+4 \lambda_{3}+4 \lambda_{4}+2 \lambda_{5}-18 \\
& \leq 6\left(r_{G_{n}}+1\right)^{2}+\left(2 r_{G_{n}}+6\right)\left(\sum_{k=0}^{5} \lambda_{k}-1\right)+2 \\
& =6\left(r_{G_{n}}+1\right)^{2}+\left(r_{G_{n}}+3\right)\left(p_{n}-12 r_{G_{n}}-6\right)+2 \\
& =-6\left(r_{G_{n}}+1\right)^{2}+\left(p_{n}-18\right)\left(r_{G_{n}}+1\right)+2 p_{n}+14 .
\end{aligned}
$$

The thesis follows by solving the inequality with respect to $r_{G_{n}}+1$ and using the definitions of $r_{n}, p_{n}$ and $\alpha_{n}$ (see (5) and Proposition 3.4).

We conclude this section with a refinement of the estimate from above on $r_{n}$.

\section{Proposition 5.12.}

$$
r_{n} \leq \rho_{n}+\mathrm{O}(1)
$$


Proof. For every $n \in \mathbb{N}, n>6$, let

$$
\tilde{\rho}_{n}:=\left\lceil\frac{\left(\alpha_{n}\right)^{2}}{3}-\frac{\left\lfloor\left(\alpha_{n}\right)^{2}-\alpha_{n}\right\rfloor}{3}-\frac{1}{3} \sqrt{\left[\left(\alpha_{n}\right)^{2}-\left\lfloor\left(\alpha_{n}\right)^{2}-\alpha_{n}\right\rfloor\right]^{2}-\left(\alpha_{n}\right)^{2}}\right\rceil .
$$

Let $H_{\tilde{\rho}_{n}}:=D_{6 \tilde{\rho}_{n}^{2}}$, and

$$
h_{n}:=\frac{p_{n}-P\left(H_{\tilde{\rho}_{n}}\right)}{4}=\frac{\left.p_{n}-6\left(2 \tilde{\rho}_{n}-1\right)\right)}{4}=\frac{p_{n}}{4}-3 \tilde{\rho}_{n}+\frac{3}{2} .
$$

Consider the hexagonal configurations $C_{c}$ given by the union of the hexagons $H_{\tilde{\rho}_{n}}$ with the "parallelograms" of height $h_{n}$ constructed on two consecutive sides of $H_{\tilde{\rho}_{n}}$. To be precise, denoting by $s_{0}^{n}, \ldots, s_{5}^{n}$ the zigzag paths passing through the sides of $H_{\tilde{\rho}_{n}}$, and setting

$$
s_{k}^{n, j}:=s_{k}^{n}+j \mathbf{e}_{k}, \quad k=0, \ldots, 5,
$$

for every $n \in \mathbb{N}$, define the set $C_{c}$ to be the portion of $\mathcal{L}$ enclosed by the zigzag paths $s_{0}^{n}, s_{1}^{n, h_{n}}, s_{2}^{n, h_{n}}, s_{3}^{n}, s_{4}^{n}, s_{5}^{n}$.

By construction, the perimeter of $C_{c}$ satisfies

$$
P\left(C_{c}\right)=p_{n} .
$$

We claim that for $n$ big enough there exists a ground state $G_{n}$ such that $H_{\tilde{\rho}_{n}} \subseteq$ $G_{n} \subseteq C_{c}$, and $\left|C_{c} \backslash G_{n}\right| \leq 2 \tilde{\rho}_{n}-1$. Indeed,

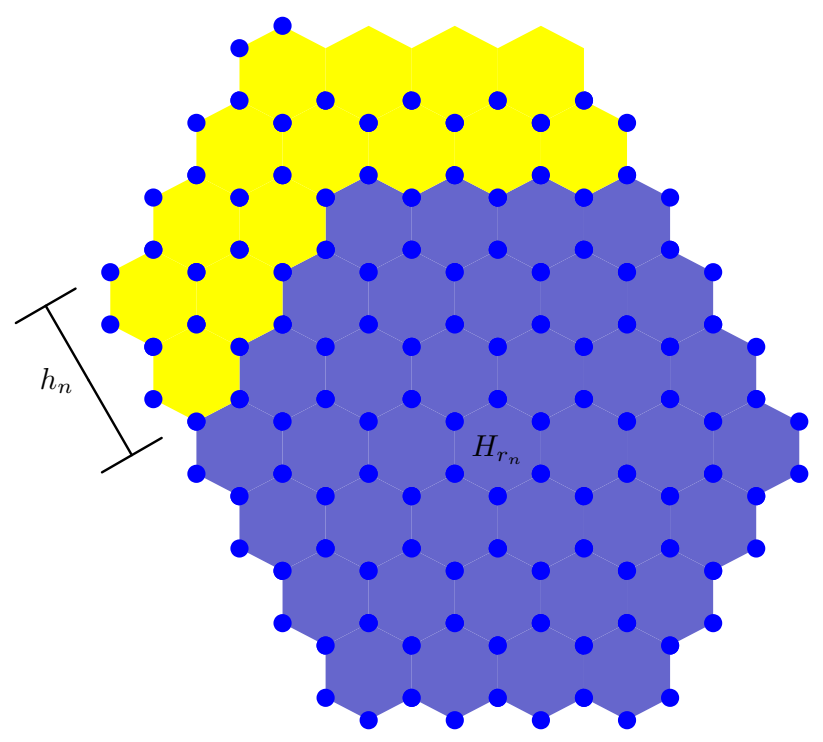

Figure 8 . In the figure above, $n=120, \tilde{\rho}_{n}=4$ and $h_{n}=2$. The configuration $C_{c}$ is defined as the union of $H_{\tilde{\rho}_{n}}$ with the two yellow parallelograms of height $h_{n}$, constructed on the sides of $H_{\tilde{\rho}_{n}}$. The ground state $G_{n}$ (given by the collection of the blue atoms) satisfies $H_{\tilde{\rho}_{n}} \subseteq G_{n} \subseteq C_{c}$, and $\left|C_{c} \backslash G_{n}\right| \leq 2 \tilde{\rho}_{n}-1$.

$$
\begin{aligned}
& \left|C_{c}\right|=\left|H_{\tilde{\rho}_{n}}\right|+4 \tilde{\rho}_{n} h_{n}=6 \tilde{\rho}_{n}^{2}+\tilde{\rho}_{n}\left[p_{n}-6\left(2 \tilde{\rho}_{n}-1\right)\right] \\
& \quad=-6 \tilde{\rho}_{n}^{2}+\left(p_{n}+6\right) \tilde{\rho}_{n} .
\end{aligned}
$$


A direct computation shows that

$$
6 s^{2}-\left(p_{n}+6\right) s+n \leq 0
$$

for every $s$ satisfying

$$
\begin{aligned}
s \in & {\left[\frac{\left(\alpha_{n}\right)^{2}}{3}-\frac{\left\lfloor\left(\alpha_{n}\right)^{2}-\alpha_{n}\right\rfloor}{3}-\frac{1}{3} \sqrt{\left[\left(\alpha_{n}\right)^{2}-\left\lfloor\left(\alpha_{n}\right)^{2}-\alpha_{n}\right\rfloor\right]^{2}-\left(\alpha_{n}\right)^{2}},\right.} \\
& \left.\frac{\left(\alpha_{n}\right)^{2}}{3}-\frac{\left\lfloor\left(\alpha_{n}\right)^{2}-\alpha_{n}\right\rfloor}{3}+\frac{1}{3} \sqrt{\left[\left(\alpha_{n}\right)^{2}-\left\lfloor\left(\alpha_{n}\right)^{2}-\alpha_{n}\right\rfloor\right]^{2}-\left(\alpha_{n}\right)^{2}}\right],
\end{aligned}
$$

whereas

$$
6 s^{2}-\left(4+p_{n}\right) s+n-1 \geq 0
$$

for every $s \in \mathbb{R}$ such that

$$
\begin{aligned}
s \leq & -\frac{1}{6}+\frac{\left(\alpha_{n}\right)^{2}}{3}-\frac{\left\lfloor\left(\alpha_{n}\right)^{2}-\alpha_{n}\right\rfloor}{3} \\
& -\frac{1}{3} \sqrt{\left[\left(\alpha_{n}\right)^{2}-\left\lfloor\left(\alpha_{n}\right)^{2}-\alpha_{n}\right\rfloor\right]^{2}-\left(\alpha_{n}\right)^{2}-\frac{1}{4}-p_{n}}
\end{aligned}
$$

or

$$
\begin{aligned}
s \geq & -\frac{1}{6}+\frac{\left(\alpha_{n}\right)^{2}}{3}-\frac{\left\lfloor\left(\alpha_{n}\right)^{2}-\alpha_{n}\right\rfloor}{3} \\
& +\frac{1}{3} \sqrt{\left[\left(\alpha_{n}\right)^{2}-\left\lfloor\left(\alpha_{n}\right)^{2}-\alpha_{n}\right\rfloor\right]^{2}-\left(\alpha_{n}\right)^{2}-\frac{1}{4}-p_{n}} .
\end{aligned}
$$

In particular, (41) and (42) hold for $s=\tilde{\rho}_{n}$, yielding

$$
0 \leq\left|C_{c}\right|-n \leq 2 \tilde{\rho}_{n}-1 .
$$

The claim follows by (43), and by observing that by the definition of $C_{c}$ it is possible to remove up to $2 \tilde{\rho}_{n}-1$ points from $C_{c} \backslash H_{\tilde{\rho}_{n}}$ without changing the perimeter of the configuration. In particular, $H_{G_{n}}=H_{\tilde{\rho}_{n}}$. The thesis is thus a direct consequence of (5), (38), and (39).

\section{Sharp convergence to the WulfF shape}

In this section we prove that as the number $n$ of atoms tends to infinity, ground states differ from a hexagonal Wulff shape by at most $\mathrm{O}\left(n^{3 / 4}\right)$ points and we show that this estimate is sharp. The proof strategy consists in exploiting Proposition 5.11 to deduce an upper bound on the number of points belonging to $G_{n}$ but not to the $n$-Wulff shape $W_{n}$.

Let $W$ be the regular hexagon defined as the convex hull of the vectors

$$
\left\{ \pm \frac{1}{\sqrt{6}} t_{1}, \pm \frac{1}{\sqrt{6}} t_{2}, \pm \frac{1}{\sqrt{6}}\left(t_{2}-t_{1}\right)\right\}
$$

and let $\chi_{W}$ be its characteristic function. Denote by $\mu$ the measure

$$
\mu:=\frac{4}{3 \sqrt{3}} \chi_{W} .
$$


6.1. Proof of Theorem 1.2. We subdivide the proof into two steps.

Step 1: Let $G_{n}$ be a ground state. Without loss of generality, assume that $n>6$, and hence, by Proposition 5.1, that the maximal hexagon $H_{G_{n}}$ is not degenerate and $r_{n} \geq 1$. Let $q_{n} \in \mathcal{L}$ be such that $H_{G_{n}}=D_{6 r_{G_{n}}^{2}}+q_{n}$. We claim that

$$
d_{\mathcal{H}}\left(G_{n}^{\prime}, W_{n}\right) \leq \mathrm{O}\left(n^{1 / 4}\right)
$$

and

$$
\left|G_{n}^{\prime} \backslash W_{n}\right|=K_{n} n^{3 / 4}+\mathrm{o}\left(n^{3 / 4}\right)
$$

where

$$
G_{n}^{\prime}:=G_{n}-q_{n}
$$

and

$$
K_{n}:=\frac{4 \alpha_{n}}{3 n^{3 / 4}} \sqrt{\left(\left(\alpha_{n}\right)^{2}-\left\lfloor\left(\alpha_{n}\right)^{2}-\alpha_{n}\right\rfloor\right)^{2}-\left(\alpha_{n}\right)^{2}},
$$

with $\alpha_{n}=\sqrt{3 n / 2}$.

Indeed, we first observe that

$$
d_{\mathcal{H}}\left(G_{n}, H_{G_{n}}\right) \leq \max _{i=0, \ldots, 5} \lambda_{i} .
$$

In view of (32) and of Proposition 5.11, we obtain the upper bounds

$$
\begin{aligned}
& d_{\mathcal{H}}\left(G_{n}, H_{G_{n}}\right) \leq 2\left(\alpha_{n}^{2}\right)-2\left\lfloor\left(\alpha_{n}\right)^{2}-\alpha_{n}\right\rfloor-6 \rho_{n} \\
& \quad \leq 18+2 \sqrt{\left[\left(\alpha_{n}\right)^{2}-\left\lfloor\left(\alpha_{n}\right)^{2}-\alpha_{n}\right\rfloor\right]^{2}-\left(\alpha_{n}\right)^{2}+39},
\end{aligned}
$$

and

$$
d_{\mathcal{H}}\left(H_{G_{n}^{\prime}}, W_{n}\right) \leq r_{G_{n}}-r_{n} \leq \frac{2}{3} \sqrt{\left[\left(\alpha_{n}\right)^{2}-\left\lfloor\left(\alpha_{n}\right)^{2}-\alpha_{n}\right\rfloor\right]^{2}-\left(\alpha_{n}\right)^{2}+39} .
$$

On the other hand, Propositions 5.11 and 5.12 yield the equality

$$
\begin{aligned}
&\left|G_{n}^{\prime} \backslash W_{n}\right|=n-6 r_{n}^{2} \\
&=n-6 \rho_{n}^{2}-6\left(r_{n}+\rho_{n}\right)\left(r_{n}-\rho_{n}\right) \\
&=n-6 \rho_{n}^{2}+\mathrm{O}\left(n^{1 / 2}\right) \\
&=n-6\left(\frac{\alpha_{n}}{3}+\frac{\left(\alpha_{n}\right)^{2}-\alpha_{n}}{3}-\frac{\left\lfloor\left(\alpha_{n}\right)^{2}-\alpha_{n}\right\rfloor}{3}-3\right. \\
&\left.-\frac{1}{3} \sqrt{\left[\left(\alpha_{n}\right)^{2}-\left\lfloor\left(\alpha_{n}\right)^{2}-\alpha_{n}\right\rfloor\right]^{2}-\left(\alpha_{n}\right)^{2}+39}\right)^{2}+\mathrm{o}\left(n^{3 / 4}\right) \\
&= n-6\left(\frac{\left(\alpha_{n}\right)^{2}}{9}-\frac{2 \alpha_{n}}{9} \sqrt{\left[\left(\alpha_{n}\right)^{2}-\left\lfloor\left(\alpha_{n}\right)^{2}-\alpha_{n}\right\rfloor\right]^{2}-\left(\alpha_{n}\right)^{2}+39}\right)+\mathrm{o}\left(n^{3 / 4}\right) \\
&= \frac{4 \alpha_{n}}{3} \sqrt{\left[\left(\alpha_{n}\right)^{2}-\left\lfloor\left(\alpha_{n}\right)^{2}-\alpha_{n}\right\rfloor\right]^{2}-\left(\alpha_{n}\right)^{2}}+\mathrm{o}\left(n^{3 / 4}\right) .
\end{aligned}
$$

Claims (44) and (45) follow now by the definition of $\alpha_{n}$ and by the observation that

$$
\sqrt{\left[\left(\alpha_{n}\right)^{2}-\left\lfloor\left(\alpha_{n}\right)^{2}-\alpha_{n}\right\rfloor\right]^{2}-\left(\alpha_{n}\right)^{2}}=\sqrt{2 \eta_{n} \alpha_{n}+\eta_{n}^{2}} \leq 1+\sqrt{\alpha_{n}}=\mathrm{O}\left(n^{1 / 4}\right),
$$

where $\eta_{n}:=\left(\alpha_{n}\right)^{2}-\alpha_{n}-\left\lfloor\left(\alpha_{n}\right)^{2}-\alpha_{n}\right\rfloor$.

Step 2: Step 1 yields the equality

$$
\left\|\mu_{G_{n}^{\prime}}-\mu_{W_{n}}\right\|=\frac{\left|G_{n}^{\prime} \triangle W_{n}\right|}{n}=K_{n} n^{-1 / 4}+\mathrm{o}\left(n^{-1 / 4}\right),
$$


where $\mu_{G_{n}^{\prime}}$ and $\mu_{W_{n}}$ are the empirical measures associated to $G_{n}^{\prime}$ and $W_{n}$, respectively (see (8)). Let $\mu_{n}:=\mu_{W_{n}}$.

For every $x_{i} \in W_{n}$, denote by $\Omega_{i}$ its Voronoi cell in $\mathcal{L}$, that is the equilateral triangle centered in $x_{i}$, of side $\sqrt{3}$ and with edges orthogonal to the three lattice directions. Finally, define $\Omega_{i}^{n}$ as the set

$$
\Omega_{i}^{n}:=\left\{x / \sqrt{n}: x \in \Omega_{i}\right\} .
$$

Let $\varphi \in W^{1, \infty}\left(\mathbb{R}^{2}\right)$. Since

$$
\left\|\frac{x_{i}}{\sqrt{n}}-x\right\|_{L^{\infty}\left(\Omega_{i}^{n}\right)} \leq \sqrt{\frac{3}{n}}
$$

and

we obtain

$$
\mathcal{L}^{2}\left(\left(\cup_{i=1}^{6 r_{n}^{2}} \Omega_{i}^{n}\right) \Delta W\right)=\mathrm{o}\left(n^{-1 / 4}\right),
$$

$$
\begin{aligned}
& \left|\int_{\mathbb{R}^{2}} \varphi d \mu_{n}-\int_{\mathbb{R}^{2}} \varphi d \mu\right|=\left|\frac{1}{n} \sum_{i=1}^{6 r_{n}^{2}} \varphi\left(\frac{x_{i}}{\sqrt{n}}\right)-\frac{4}{3 \sqrt{3}} \int_{W} \varphi d x\right| \\
& \quad=\frac{4}{3 \sqrt{3}}\left|\sum_{i=1}^{6 r_{n}^{2}} \varphi\left(\frac{x_{i}}{\sqrt{n}}\right) \mathcal{L}^{2}\left(\Omega_{i}^{n}\right)-\int_{W} \varphi d x\right| \\
& \quad \leq \frac{4}{3 \sqrt{3}}\left|\sum_{i=1}^{6 r_{n}^{2}} \int_{\Omega_{i}^{n}}\left(\varphi\left(\frac{x_{i}}{\sqrt{n}}\right)-\varphi(x)\right) d x\right|+\frac{4}{3 \sqrt{3}}\|\varphi\|_{L^{\infty}\left(\mathbb{R}^{2}\right)} \mathcal{L}^{2}\left(\left(\cup_{i=1}^{6 r_{n}^{2}} \Omega_{i}^{n}\right) \Delta W\right) \\
& \quad \leq \frac{4}{3 \sqrt{3}}\|\nabla \varphi\|_{L^{\infty}\left(\mathbb{R}^{2} ; \mathbb{R}^{2}\right)} \sum_{i=1}^{6 r_{n}^{2}} \int_{\Omega_{i}^{n}}\left|\frac{x_{i}}{\sqrt{n}}-x\right| d x+\frac{4}{3 \sqrt{3}}\|\varphi\|_{L^{\infty}\left(\mathbb{R}^{2}\right)} \mathcal{L}^{2}\left(\left(\cup_{i=1}^{6 r_{n}^{2}} \Omega_{i}^{n}\right) \Delta W\right) \\
& \quad \leq \frac{4}{3 \sqrt{n}}\|\nabla \varphi\|_{L^{\infty}\left(\mathbb{R}^{2} ; \mathbb{R}^{2}\right)} \mathcal{L}^{2}\left(\cup_{i=1}^{6 r_{n}^{2}} \Omega_{i}^{n}\right)+\frac{4}{3 \sqrt{3}}\|\varphi\|_{L^{\infty}\left(\mathbb{R}^{2}\right)} \mathcal{L}^{2}\left(\left(\cup_{i=1}^{6 r_{n}^{2}} \Omega_{i}^{n}\right) \Delta W\right) \\
& \quad=\|\varphi\|_{W^{1, \infty}\left(\mathbb{R}^{2}\right) \mathrm{O}\left(n^{-1 / 4}\right) .}
\end{aligned}
$$

Denoting by $G_{n}^{\prime}$ the set $G_{n}^{\prime}:=G_{n}-q_{n}$, and by $\mu_{G_{n}^{\prime}}$ its associated empirical measure, inequalities (48) and (49) yield

$$
\mu_{\left(G_{n}\right)^{\prime}} \rightarrow^{*} \mu, \quad \text { weakly* in } M_{b}\left(\mathbb{R}^{2}\right),
$$

and

$$
\left\|\mu_{\left(G_{n}\right)^{\prime}}-\mu\right\|_{\mathrm{F}}=K_{n} n^{-1 / 4}+\mathrm{o}\left(n^{-1 / 4}\right) .
$$

We notice that $K_{n}=0$ for every $n \in \mathbb{N}$ such that $n=6 k^{2}$ for some $k \in \mathbb{N}$. This reflects the fact that for those $n$ the daisy $D_{n}$ is the unique ground state, whose maximal hexagon is the daisy itself.

In view of the definition of $\alpha_{n}$, a direct computation shows that

$$
K_{n}=\frac{2^{7 / 4}}{3^{1 / 4}} \sqrt{\left(\frac{3 n}{2}-\sqrt{\frac{3 n}{2}}\right)-\left(\left\lfloor\frac{3 n}{2}-\sqrt{\frac{3 n}{2}}\right)\right\rfloor}+\mathrm{o}(1) .
$$

Hence, in particular,

$$
\limsup _{n \rightarrow+\infty} K_{n} \leq \frac{2^{7 / 4}}{3^{1 / 4}}=K
$$


This completes the proof of Theorem 1.2.

6.2. Proof of Theorem 1.3. The proof consists in finding a sequence $\left\{n_{i}\right\}, i \in \mathbb{N}$, such that

$$
K_{n_{i}} \rightarrow K
$$

as $i \rightarrow+\infty$. Indeed, in view of (45), (48), (51), (52), for every $\left\{n_{i}\right\}$ verifying (52), and for every sequence of ground states $\left\{G_{n_{i}}\right\}$, there exist suitable translations $\left\{G_{n_{i}}^{\prime}\right\}$ such that

and

$$
\begin{aligned}
\left|G_{n_{i}}^{\prime} \backslash W_{n_{i}}\right| & =K n_{i}^{3 / 4}+\mathrm{o}\left(n_{i}^{3 / 4}\right), \\
\left\|\mu_{G_{n_{i}}^{\prime}}-\mu_{W_{n_{i}}}\right\| & =K n_{i}^{-1 / 4}+\mathrm{o}\left(n_{i}^{-1 / 4}\right),
\end{aligned}
$$

$$
\left\|\mu_{G_{n_{i}}^{\prime}}-d \chi_{W}\right\|_{\mathrm{F}}=K n_{i}^{-1 / 4}+\mathrm{o}\left(n^{-1 / 4}\right) .
$$

A possible choice is to consider

$$
n_{i}:=2+6 i^{2} .
$$

In fact we have

$$
\frac{3 n_{i}}{2}-\sqrt{\frac{3 n_{i}}{2}}=9 i^{2}+3-\sqrt{9 i^{2}+3}=9 i^{2}+3-3 i-\frac{1}{i\left(1+\sqrt{1+\frac{1}{3 i^{2}}}\right)},
$$

and hence

$$
\left(\frac{3 n_{i}}{2}-\sqrt{\frac{3 n_{i}}{2}}\right)-\left\lfloor\left(\frac{3 n_{i}}{2}-\sqrt{\frac{3 n_{i}}{2}}\right)\right\rfloor=1-\frac{1}{i\left(1+\sqrt{1+\frac{1}{3 i^{2}}}\right)} \rightarrow 1
$$

as $i \rightarrow+\infty$, which in turn yields (52). This completes the proof of Theorem 1.3.

Before closing this section let us comment on the fact that, as a byproduct of our construction, we also obtain sharp estimates on the distance of any sequence $\left\{G_{n}\right\}$ of (translated) ground states from the $n$-Wulff shape, in terms of the constant $K_{n}$ defined in (46) (see (45), (48), and (51)). Let us finally stress the nonuniqueness of the $n$-dimentional Wulff shape $W_{n}$ : any zigzag hexagon $D_{6} \tilde{r}_{n}^{2}$, with radius $\tilde{r}_{n}=$ $r_{n}+\mathrm{O}(1)$ (e.g. $\left.\tilde{r}_{n}=\tilde{\rho}_{n}\right)$ would infact lead to the same sharp results.

\section{ACKNOWLEDGEMENTS}

The authors were supported by the Austrian Science Fund (FWF) project P 27052-N25. This work has been funded by the Vienna Science and Technology Fund (WWTF) through project MA14-009. Partial support by the Wolfgang Pauli Institute under the thematic project Crystals, Polymers, Materials is also acknowledged. E.D. and P.P. thank the Center for Nonlinear Analysis (NSF Grant No. DMS-0635983), where part of this research was carried out. E.D. was partially funded by the National Science Foundation (NSF) under Grant No. DMS- 0905778, and under the PIRE Grant No. OISE-0967140. E.D. is a member of the INdAMGNAMPA Project 2015 Critical phenomena in the mechanics of materials: a variational approach. 


\section{REFERENCES}

[1] V. Artyukhov, Y. Hao, R.S. Ruoff, B.I. Yakobson. Breaking of symmetry in graphene growth on metal substrates. Phys. Rev. Lett. 114 (2015), 115502.

[2] Y. Au Yeung, G. Friesecke, B. Schmidt. Minimizing atomic configurations of short range pair potentials in two dimensions: crystallization in the Wulff-shape. Calc. Var. Partial Differential Equations, 44 (2012), 81-100.

[3] L. Barletti. Hydrodynamic equations for electrons in graphene obtained from the maximum entropy principle. J. Math. Phys. 55 (2014), 083303.

[4] X. Blanc, M. Lewin. The crystallization conjecture: a review. EMS Surv. Math. Sci. 2 (2015), 255-306.

[5] P.S. Branicio, M.H. Jhon, C.K. Gan, D.J. Srolovitz. Properties on the edge: graphene edge energies, edge stresses, edge warping, and the Wulff shape of graphene flakes. Modelling Simul. Mater. Sci. Eng. 19 (2011), 054002.

[6] V.D. Camiola, V. Romano. Hydrodynamical model for charge transport in graphene. J. Stat. Phys. 157 (2014), 1114-1137.

[7] G. Dal Maso. An introduction to $\Gamma$-convergence. Boston, Birkhäuser, 1993.

[8] C. Davini. Homogenization of a graphene sheet. Contin. Mech. Thermodyn. 26 (2014), 95113.

[9] E. Davoli, P. Piovano, U. Stefanelli. Sharp $n^{3 / 4}$ law for the minimizers of the edgeisoperimetric problem on the triangular lattice. Submitted (2015).

[10] A. Dobry, O. Fojón, M. Gadella, L.P. Lara. Some numerical estimations of energy levels on a model for a graphene ribbon in a magnetic field. Appl. Math. Comput. 235 (2014), 8-16.

[11] C.T.J. Dodson. A model for gaussian perturbations of graphene. J. Stat. Phys. 161 (2015), 933-941.

[12] W. E, D. Li. On the crystallization of 2D hexagonal lattices. Comm. Math. Phys. 286 (2009), 1099-1140.

[13] R. El Hajj, F. Méhats. Analysis of models for quantum transport of electrons in graphene layers. Math. Models Methods Appl. Sci. 24 (2014), 2287-2310.

[14] D. El Kass, R. Monneau. Atomic to continuum passage for nanotubes: a discrete Saint-Venant principle and error estimates. Arch. Ration. Mech. Anal. 213 (2014), 25-128.

[15] B. Farmer, S. Esedoḡlu, P. Smereka. Crystallization for a Brenner-like potential. ArXiv: 1512.04489, 2015.

[16] C.L. Fefferman, M.I. Weinstein. Wave packets in honeycomb structures and two-dimensional Dirac equations. Comm. Math. Phys. 326 (2014), 251-286.

[17] L. Flatley, M. Taylor, A. Tarasov, F. Theil. Packing twelve spherical caps to maximize tangencies. J. Comput. Appl. Math. 254 (2013), 220-225.

[18] L. Flatley, F. Theil. Face-centered cubic crystallization of atomistic configurations. Arch. Ration. Mech. Anal. 218 (2015), 363-416.

[19] C.K. Gan, D.J. Srolovitz. First-principles study of graphene edge properties and flake shapes. Phys. Rev. B, 81 (2010), 125445-125453.

[20] C.S. Gardner, C. Radin. The infinite-volume ground state of the Lennard-Jones potential. J. Stat. Phys. 20 (1979), 719-724.

[21] C.O. Girit, J.C. Meyer, R. Erni, M.D. Rossel, C. Kisielowski, L. Yang, C.H. Park, M.F. Crommie, M.L. Cohen, S.G. Louie, A. Zettl. Graphene at the edge: stability and dynamics. Science, 27 (2009), 1705-1708.

[22] J.E. Graver, C. Graves, S.J. Graves. Fullerene patches II. Ars Math. Contemp. 7 (2014), 405-421.

[23] L. H. Harper. Global methods for combinatorial isoperimetric problems. Cambridge Studies in Advanced Mathematics, 90. Cambridge University Press, Cambridge, 2004.

[24] R. Heitmann, C. Radin. Ground states for sticky disks. J. Stat. Phys. 22 (1980), 281-287.

[25] P. Kerdelhué, J. Royo-Letelier. On the low lying spectrum of the magnetic Schrödinger operator with Kagomé periodicity. Rev. Math. Phys. 26 (2014), 1450020, 46.

[26] Z. Luo, S. Kim, N. Kawamoto, A.M. Rappe, A.T. Charlie Johnson. Growth mechanism of hexagonal-shape graphene flakes with zigzag edges. ACSNano, 11 (2011), 1954-1960.

[27] J. Lu, V. Moroz, C.B. Muratov. Orbital-free density functional theory of out-of-plane charge screening in graphene. J. Nonlinear Sci. 25 (2015), 1391-1430. 
[28] E. Mainini, H. Murakawa, P. Piovano, U. Stefanelli. A numerical investigation on carbonnanotube geometries. Discrete Contin. Dyn. Syst. - S, (2015), to appear.

[29] E. Mainini, H. Murakawa, P. Piovano, U. Stefanelli. Variational modeling of carbon nanotubes. In preparation, 2016.

[30] E. Mainini, P. Piovano, U. Stefanelli. Finite crystallization in the square lattice. Nonlinearity, 27 (2014), 717-737.

[31] E. Mainini, P. Piovano, U. Stefanelli. Crystalline and isoperimetric square configurations. Proc. Appl. Math. Mech. 14 (2014), 1045-1048.

[32] E. Mainini, U. Stefanelli. Crystallization in carbon nanostructures. Comm. Math. Phys. 328 (2014), 545-571.

[33] M. Makwana, R.V. Craster. Homogenisation for hexagonal lattices and honeycomb structures. Quart. J. Mech. Appl. Math. 67 (2014), 599-630.

[34] D. Monaco, G. Panati. Topological invariants of eigenvalue intersections and decrease of Wannier functions in graphene. J. Stat. Phys. 155 (2014), 1027-1071.

[35] D. Monaco, G. Panati. Symmetry and localization in periodic crystals: triviality of Bloch bundles with a fermionic time-reversal symmetry. Acta Appl. Math. 137 (2015), 185-203.

[36] C. Radin. The ground state for soft disks, J. Stat. Phys. 26 (1981), 365-373.

[37] B. Schmidt. Ground states of the 2D sticky disc model: fine properties and $N^{3 / 4}$ law for the deviation from the asymptotic Wulff-shape. J. Stat. Phys. 153 (2013), 727-738.

[38] F.H. Stillinger, T.A. Weber. Computer simulation of local order in condensed phases of silicon. Phys. Rev. B, 8 (1985), 5262-5271.

[39] J. Tersoff. New empirical approach for the structure and energy of covalent systems. Phys. Rev. B, 37 (1988), 6991-7000.

[40] F. Theil. A proof of crystallization in two dimensions. Comm. Math. Phys. 262 (2006), 209236.

[41] W.J. Ventevogel. On the configuration of a one-dimensional system of interacting atoms with minimum potential energy per atom. Phys. A, 92 (1978), 343-361.

[42] W.J. Ventevogel, B.R.A. Nijboer. On the configuration of systems of interacting atom with minimum potential energy per atom. Phys. A, 99 (1979), 565-580.

[43] H.J. Wagner. Crystallinity in two dimensions: a note on a paper of C. Radin. J. Stat. Phys. 33 (1983), 523-526.

[44] Y. Zhang, L.W. Zhang, K.M. Liew, J.L. Yu. Transient analysis of single-layered graphene sheet using the kp-Ritz method and nonlocal elasticity theory. Appl. Math. Comput. 258 (2015), 489-501.

(Elisa Davoli) Faculty of Mathematics, University of Vienna, Oskar-MorgensternPlatz 1, A-1090 Vienna, Austria

E-mail address: elisa.davoli@univie.ac.at

(Paolo Piovano) Faculty of Mathematics, University of Vienna, Oskar-MorgensternPlatz 1, A-1090 Vienna, Austria

E-mail address: paolo.piovano@univie.ac.at

(Ulisse Stefanelli) Faculty of Mathematics, University of Vienna, Oskar-MorgensternPlatz 1, A-1090 Vienna, Austria, and Istituto di Matematica Applicata e Tecnologie Informatiche "E. Magenes" - CNR, v. Ferrata 1, I - 27100 Pavia, Italy

E-mail address: ulisse.stefanelli@univie.ac.at 